\title{
EULER SCHEMES AND HALF-SPACE APPROXIMATION FOR THE SIMULATION OF DIFFUSION IN A DOMAIN
}

\author{
Emanuel GoBet ${ }^{1}$
}

\begin{abstract}
This paper is concerned with the problem of simulation of $\left(X_{t}\right)_{0 \leq t \leq T}$, the solution of a stochastic differential equation constrained by some boundary conditions in a smooth domain $D$ : namely, we consider the case where the boundary $\partial D$ is killing, or where it is instantaneously reflecting in an oblique direction. Given $N$ discretization times equally spaced on the interval $[0, T]$, we propose new discretization schemes: they are fully implementable and provide a weak error of order $N^{-1}$ under some conditions. The construction of these schemes is based on a natural principle of local approximation of the domain into a half space, for which efficient simulations are available.
\end{abstract}

AMS Subject Classification. 35K20, 60-08, 60J60, 65Cxx.

Received September 3, 2001. Revised December 10, 2001.

\section{CONTEnts}

Introduction

1. Preliminaries 263

1.1. Notation 263

1.2. About the projection and the distance to the boundary 264

1.3. Green-Riemann's formula 264

2. Killed diffusions $\quad 265$

2.1. Algorithm and analysis of the error 267

\begin{tabular}{lr}
2.2. & Proofs \\
\hline
\end{tabular}

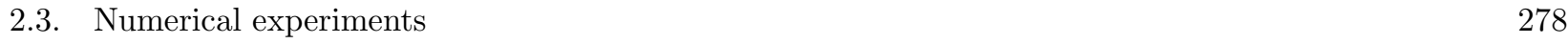

3. Reflected diffusions 279

3.1. Algorithm and analysis of the error 281

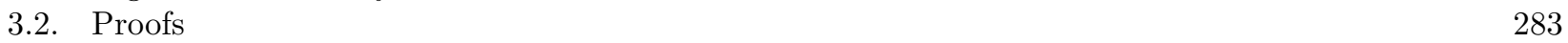

3.3. Numerical experiments $\quad 292$

4. Conclusion 293

Appendix A. Proof of Proposition 1.1 295

\begin{tabular}{l} 
References \\
\hline
\end{tabular}

Keywords and phrases: Killed diffusion, reflected diffusion, discretization schemes, rates of convergence, weak approximation, boundary value problems for parabolic PDE.

${ }^{1}$ École Polytechnique, Centre de Mathématiques Appliquées, 91128 Palaiseau Cedex, France;

e-mail: emmanuel.gobet@polytechnique.fr 


\section{INTRODUCTION}

We consider $\left(X_{t}\right)_{0 \leq t \leq T}$, a diffusion process, with boundary conditions, in a domain $D \subset \mathbb{R}^{d}$ ( $T$ is a fixed time). We will mainly deal with two situations: the process is killed when it reaches the boundary $\partial D$, or it is instantaneously reflected in some given direction. The purpose of this paper is to present and analyze some new Monte-Carlo algorithms to compute the expectation of functionals of $\left(X_{t}\right)_{0 \leq t \leq T}$.

1) Killed diffusion

In that case, we consider the process $\left(X_{t}\right)_{0 \leq t \leq T}$, solution of the stochastic differential equation (SDE in short)

$$
X_{t}=x+\int_{0}^{t} B\left(X_{s}\right) \mathrm{d} s+\int_{0}^{t} \sigma\left(X_{s}\right) \mathrm{d} W_{s}
$$

and we are interested by the functional $\mathbf{1}_{T<\tau} f\left(X_{T}\right)$ with $\tau=\inf \left\{t>0: X_{t} \notin D\right\}$, for a given function $f$.

\section{2) Reflected diffusion}

Here, the process $\left(X_{t}\right)_{0 \leq t \leq T}$ is a reflected stochastic differential equation (RSDE in short) in $D$, with oblique reflection in the direction $\gamma$, i.e. the $D$-valued process which solves

$$
X_{t}=x+\int_{0}^{t} B\left(X_{s}\right) \mathrm{d} s+\int_{0}^{t} \sigma\left(X_{s}\right) \mathrm{d} W_{s}+\int_{0}^{t} \gamma\left(X_{s}\right) \mathrm{d} k_{s},
$$

where $k_{t}$ is a local time process which increases only on $\partial D$. The functional of interest may depend both on the terminal value of $X$ and on the local time $\left(k_{t}\right)_{0 \leq t \leq T}$ : for example, we will consider $f\left(X_{T}\right)-$ $\int_{0}^{T} h\left(X_{t}\right) \mathrm{d} k_{t}$.

For the above situations, assumptions on the coefficients, the domain and the functions will be given later.

The evaluation of the expectation of such functionals is motivated by many applications. Let us give few examples. For killed diffusions, this can be related to the pricing of barrier options in finance (see e.g. [2,12]). For reflected diffusions, the computation of the expectation may contribute as in [4] and [8], to reconstruct the three dimensional brain activity via the resolution of some partial differential equations (PDE in short) with Neumann conditions. Reflected diffusions also appear as approximation models for open queuing networks in heavy traffic (see e.g. the review by Williams [33]). More generally, the expectations to evaluate are solutions of second order PDE with Dirichlet or Neumann conditions, so the procedures presented here enable to compute the corresponding solution using Monte-Carlo simulations.

For the numerical simulations of SDEs, the most standard approach consists in discretizing the time interval $[0, T]$ with a regular mesh, at times $t_{i}=i T N^{-1}$ and to approximate the SDE in an appropriate way on each interval $\left[t_{i}, t_{i+1}\right]$. When there is no boundary condition (i.e. $X_{t}=x+\int_{0}^{t} B\left(X_{s}\right) \mathrm{d} s+\int_{0}^{t} \sigma\left(X_{s}\right) \mathrm{d} W_{s}$ and we compute $\left.\mathbb{E}\left(f\left(X_{T}\right)\right)\right)$, one may simply use the Euler scheme $\left(X_{t_{i}}^{N}\right)_{0 \leq i \leq N}$ defined step by step by

$$
X_{t_{i+1}}^{N}=X_{t_{i}}^{N}+B\left(X_{t_{i}}^{N}\right)\left(t_{i+1}-t_{i}\right)+\sigma\left(X_{t_{i}}^{N}\right)\left(W_{t_{i+1}}-W_{t_{i}}\right)
$$

It is easy to implement since it requires only the simulation of $d$-independent Gaussian variables at each step, and provides a weak error of order $N^{-1}$, i.e. $\mathbb{E}\left(f\left(X_{T}\right)\right)-\mathbb{E}\left(f\left(X_{T}^{N}\right)\right)=O\left(N^{-1}\right)$ under some conditions (see $[3,32]$ and references therein).

When there are some boundary conditions in a general domain $D$, it is well known that a naive adaptation of the Euler scheme above yields in general a slower rate of convergence $N^{-1 / 2}$ (see [12] for the killing; Ref. [7] for the reflection). Nevertheless, if $D$ is a half-space, feasible improvements of the naive procedure are available using some additional Brownian laws (references and details will be given in Sects. 2 and 3)): thus, one may hope to exploit these situations where the domain is reduced to half-space, to treat the cases where $D$ is more 
general. One possibility for this, is to locally approximate the domain $D$ as a half-space, situation for which improved procedures cited above can be used: this is the basic idea of our approach.

This natural principle leads to simple and fully implementable algorithms, which have the advantage to converge at the rate $N^{-1}$ under some appropriate assumptions. Numerical experiments confirm the accuracy of these new procedures. The paper is organized as follows:

1. in Section 1, we introduce notation used in the paper, and recall some standard results from differential geometry and analysis;

2. in Section 2, we deal with killed diffusions. First, we briefly recall backgrounds from the literature; then we describe the new algorithm, state the convergence results and give their proofs. Some numerical experiments confirm the accuracy of the method;

3. in Section 3, we consider the case of the reflection. After a review of literature results, we present the numerical procedure. Then, we state the convergence results, give their proofs, and illustrate the method by some numerical tests.

Sections 2 and 3 can be red in a rather independent way: nevertheless, some technical arguments are common to both parts.

\section{Preliminaries}

\subsection{Notation}

In the following, we consider a domain $D \subset \mathbb{R}^{d}$, i.e. an open connected set, which satisfies the following hypothesis:

Assumption (D). The boundary $\partial D$ is bounded and of class $C^{5}$.

For some $x \in \partial D$, let denote by $n(x)$ the unit inward normal vector at $x$.

For sets $\Lambda$ and $\Lambda^{\prime}$ in $\mathbb{R}^{d}$, for $z \in \mathbb{R}^{d}, d(z, \Lambda)$ stands for the Euclidean distance between $z$ and $\Lambda, d\left(\Lambda, \Lambda^{\prime}\right)$ for the distance between $\Lambda$ and $\Lambda^{\prime}$.

For $r \geq 0$, set $V_{\partial D}(r):=\left\{z \in \mathbb{R}^{d}: d(z, \partial D) \leq r\right\}$ and $D(r):=\left\{z \in \mathbb{R}^{d}: d(z, D) \leq r\right\}$.

For smooth functions $g(t, x)$, we denote by $\partial_{x}^{\alpha} g(t, x)$ the derivative of $g$ w.r.t. $x$ according to the multiindex $\alpha$, whereas time derivatives of $g$ are denoted by $\partial_{t} g(t, x), \partial_{t}^{2} g(t, x), \ldots$ The notation $\nabla g(t, x)$ stands for the usual gradient w.r.t. $x$.

If $\left(V_{t}\right)_{t \geq 0}$ is a process taking its values in $\mathbb{R}^{d},\left(V_{i, t}\right)_{t \geq 0}$ will denote its $i$-th coordinate. The transposition of the matrix $U$ is denoted by $U^{*}$.

As usual, the index $x$ in $\mathbb{E}_{x}$ and $\mathbb{P}_{x}$ refers to the initial value of some Markov process for which we compute the expectation or the probability: this will be clear from the context.

About the discretization. We will consider $N$ discretization times in the interval $[0, T]$, regularly spaced: we denote them by $t_{i}=i T / N$. The function $\varphi$ is defined on $[0, T)$ by:

$$
\varphi(t)=t_{i} \quad \text { if } \quad t_{i} \leq t<t_{i+1} .
$$

About the floating constants. We will keep the same notation $K(T)$ for all finite, non-negative and nondecreasing functions, independent of the number of time steps $N$, which will appear in proofs (i.e. they depend on $D$, the coefficients which define the process $X$, the functions involved in the expectation of interest and so on). 
We reserve the notation $c$ and $c^{\prime}$ for some positive constants, independent of $N \ldots$ as for $K(T)$ and also independent of $T$.

A quantity will be denoted by $O_{\text {pol }}(N)$ if it is bounded by $K_{k}(T) N^{-k}$ for any $k \geq 0$. The constant $K_{k}(T)$ is uniform in all parameters from the context, e.g. $x, t \in[0, T], N \ldots$

\subsection{About the projection and the distance to the boundary}

Here, we recall some classical results from differential geometry, about the functions "distance" to $\partial D$ or $D$ and "projection" on $\partial D$ or $D$, parallel to some vector field $\gamma$ defined on $\partial D$. We assume that this vector field is normalized to $1:\|\gamma(x)\|=1$ for $x \in \partial D$.

We will consider that $\gamma$ is smooth, inward and uniformly non tangent to $\partial D$.

Assumption $(\Gamma) . \gamma$ is of class $C^{4}$ and satisfies

$$
\forall x \in \partial D \quad \gamma(x) \cdot n(x) \geq \rho_{0}>0
$$

The following proposition brings together the main geometric materials which will be used in the paper: its proof is given in Appendix.

Proposition 1.1. Assume (D) and $(\Gamma)$. There is a constant $R>0$ such that:

i) for any $x \in V_{\partial D}(R)$, there are unique $s=\pi_{\partial D}^{\gamma}(x) \in \partial D$ and $F^{\gamma}(x) \in \mathbb{R}$ such that:

$$
x=\pi_{\partial D}^{\gamma}(x)+F^{\gamma}(x) \gamma\left(\pi_{\partial D}^{\gamma}(x)\right)
$$

ii) the function $x \longmapsto \pi_{\partial D}^{\gamma}(x)$ is called the projection of $x$ on $\partial D$ parallel to $\gamma$ : this is a $C^{4}$-function on $V_{\partial D}(R)$

iii) the function $x \longmapsto F^{\gamma}(x)$ is called the algebraic distance of $x$ to $\partial D$ parallel to $\gamma$ : this is a $C^{4}$-function on $V_{\partial D}(R)$. One has $F^{\gamma}>0$ on $V_{\partial D}(R) \cap D, F^{\gamma}<0$ on $V_{\partial D}(R) \cap \bar{D}^{c}, F^{\gamma}=0$ on $\partial D$ : we extend $F^{\gamma}$ into a $C_{b}^{4}\left(\mathbb{R}^{d}, \mathbb{R}\right)$ function, with the conditions $F^{\gamma}>0$ on $D$ and $F^{\gamma}<0$ on $\bar{D}^{c}$;

iv) on $V_{\partial D}(R)$, the projection of $x$ on $D$ parallel to $\gamma$ is given by $\pi_{D}^{\gamma}(x)=\pi_{\partial D}^{\gamma}(x)+\left(F^{\gamma}\right)^{+}(x) \gamma\left(\pi_{\partial D}^{\gamma}(x)\right)$, and its distance to $D$ is defined by $\left(F^{\gamma}\right)^{-}(x)$;

$\mathrm{v})$ the above extensions for $F^{\gamma}$ and $F^{n}$ can be performed in a way such that the functions $F^{\gamma}$ and $F^{n}$ are in some sense equivalent, i.e. for some constant $c_{1}>1$, one has

$$
\frac{1}{c_{1}}\left|F^{n}(x)\right| \leq\left|F^{\gamma}(x)\right| \leq c_{1}\left|F^{n}(x)\right| \quad \forall x \in \mathbb{R}^{d}
$$

vi) for $x \in \partial D$, one has $\nabla F^{\gamma}(x)=\frac{n}{n \cdot \gamma}(x)$.

\subsection{Green-Riemann's formula}

For some smooth functions $B$ and $\sigma$, consider $L$ the second order operator defined on $C^{2}$ functions by

$$
L u(x)=\sum_{i=1}^{d} B_{i}(x) \partial_{i} u(x)+\frac{1}{2} \sum_{i=1}^{d} \sum_{j=1}^{d}\left(\sigma \sigma^{*}(x)\right)_{i, j} \partial_{i, j}^{2} u(x)
$$


Its formal adjoint $L^{*}$ is given by $L^{*} u(x)=-\sum_{i=1}^{d} \partial_{i}\left[B_{i}(x) u(x)\right]+\frac{1}{2} \sum_{i=1}^{d} \sum_{j=1}^{d} \partial_{i, j}^{2}\left[\left(\sigma \sigma^{*}(x)\right)_{i, j} u(x)\right]$. Then, Green-Riemann's formula (see [24] Sect. 1.6) implies that, for $u$ and $v$ two $C^{2}$ functions, one has

$$
\begin{aligned}
\int_{D} L^{*} v u \mathrm{~d} x= & \int_{D} L u v \mathrm{~d} x+\frac{1}{2} \sum_{i, j} \int_{\partial D} v \partial_{i} u\left[\sigma \sigma^{*}\right]_{i, j} n_{j} \mathrm{~d} s \\
& +\sum_{i} \int_{\partial D} u v B_{i} n_{i} \mathrm{~d} s-\frac{1}{2} \sum_{i, j} \int_{\partial D} u \partial_{i}\left\{\left[\sigma \sigma^{*}\right]_{i, j} v\right\} n_{i} \mathrm{~d} s .
\end{aligned}
$$

Such a formula is valid for smooth domain $D$, satisfying e.g. Assumptions (D) and ( $\Gamma$ ), but also for half-space up to considering some appropriate decay conditions at infinity on $u, v$ and their derivatives, to ensure that all above integrals are well defined.

We now recall what is the conormal direction.

Definition 1.2 (Conormal direction). The vector $\gamma^{c}$ associated to the domain $D$ and the diffusion coefficient $\sigma$ is conormal if

$$
\forall s \in \partial D \quad \gamma^{c}(s)=\frac{\sigma \sigma^{*}(s) n(s)}{\left\|\sigma \sigma^{*}(s) n(s)\right\|},
$$

where we assume that $\sigma \sigma^{*}(s) n(s) \neq 0$.

Furthermore, for some vector field $\gamma$, which is assumed to be non tangent to $\partial D$ (it may satisfy for instance the assumption $(\Gamma))$, put

$$
a^{\gamma}=\left\|\sigma \sigma^{*} n\right\| \frac{n \cdot \gamma^{c}}{n \cdot \gamma}=\frac{n \cdot \sigma \sigma^{*} n}{n \cdot \gamma}
$$

Then, the equality (4) can be rewritten as

$$
\int_{D} L^{*} v u \mathrm{~d} x=\int_{D} L u v \mathrm{~d} x+\int_{\partial D} \frac{1}{2} a^{\gamma} v \nabla u \cdot \gamma \mathrm{d} s+\int_{\partial D} u\left(-\frac{1}{2} a^{\gamma^{*}} \nabla v \cdot \gamma^{*}+b v\right) \mathrm{d} s,
$$

for some non tangent vector field $\gamma^{*}$ and some function $b$, depending on $B, \sigma$ and $D$. Detailed expression of these quantities are available (see [24] Sect. 1.6, [9] Sect. 2.5), but will not be needed in the following. Let us mention that when $\gamma=\gamma^{c}$, one has $\gamma^{*}=\gamma^{c}$; thus, in general, the case of conormal direction enjoys some simplest analysis. In the PDE terminology, this case is associated to the so-called Neumann problem or second boundary value problem, whereas if $\gamma \neq \gamma^{c}$, this is the regular oblique derivative problem or third boundary value problem.

\section{Killed Diffusions}

In this section, we consider the $\mathbb{R}^{d}$-valued diffusion process $\left(X_{t}\right)_{t \geq 0}$, defined by

$$
X_{t}=x+\int_{0}^{t} B\left(X_{s}\right) \mathrm{d} s+\int_{0}^{t} \sigma\left(X_{s}\right) \mathrm{d} W_{s},
$$

where $\left(W_{t}\right)_{t \geq 0}$ is a $d$-dimensional Brownian motion. Let $L$ be its infinitesimal generator defined by (3). Denote by $\tau:=\inf \left\{t>0: X_{t} \notin D\right\}$, its first exit time from the domain $D$ (we implicitly assume $x \in D$ ). We are interested in computing $\mathbb{E}_{x}\left[\mathbf{1}_{T<\tau} f\left(X_{T}\right)\right]$, for a given measurable function $f$, and a fixed time $T$, using Monte-Carlo simulations. 
For this, the simplest method (but far to be accurate) is to use its discrete Euler scheme $\left(X_{t_{i}}^{N}\right)_{0 \leq i \leq N}$ with time step $T / N$, defined by

$$
\left\{\begin{array}{ccc}
X_{0}^{N} & =x \\
X_{t_{i+1}}^{N} & =X_{t_{i}}^{N}+B\left(X_{t_{i}}^{N}\right) T / N+\sigma\left(X_{t_{i}}^{N}\right)\left(W_{t_{i+1}}-W_{t_{i}}\right) .
\end{array}\right.
$$

Let $\tau_{d}^{N}:=\inf \left\{t_{i}: X_{t_{i}}^{N} \notin D\right\}$ be its first exit time from $D$ : the random variable $\mathbf{1}_{T<\tau_{d}^{N}} f\left(X_{T}^{N}\right)$ is straightforward to simulate (it involves only the values $\left(X_{t_{i}}^{N}\right)_{0 \leq i \leq N}$ ), but it is a rough approximation of $\mathbf{1}_{T<\tau} f\left(X_{T}\right)$ in a weak sense. Indeed, under some conditions, the author [12] has proved that

$$
\mathbb{E}_{x}\left[\mathbf{1}_{T<\tau_{d}^{N}} f\left(X_{T}^{N}\right)-\mathbf{1}_{T<\tau} f\left(X_{T}\right)\right]=O\left(N^{-1 / 2}\right)
$$

and the above estimate is tight.

To improve the procedure, one can interpolate the previous discrete time process (8) into a continuous Euler scheme $\left(X_{t}^{N}\right)_{0 \leq t \leq T}$ by setting

$$
\text { for } t \in\left[t_{i}, t_{i+1}\right) \quad X_{t}^{N}=X_{t_{i}}^{N}+B\left(X_{t_{i}}^{N}\right)\left(t-t_{i}\right)+\sigma\left(X_{t_{i}}^{N}\right)\left(W_{t}-W_{t_{i}}\right)
$$

and $\tau_{c}^{N}:=\inf \left\{t: X_{t}^{N} \notin D\right\}$. The weak approximation of $\mathbf{1}_{T<\tau} f\left(X_{T}\right)$ by $\mathbf{1}_{T<\tau_{d}^{N}} f\left(X_{T}^{N}\right)$ is more accurate than before since it is of order $N^{-1}$ (see [12]), but the problem is how to simulate the random variable $Z^{N}:=$ $\mathbf{1}_{T<\tau_{c}^{N}} f\left(X_{T}^{N}\right)$. Actually, once simulated $\left(X_{t_{i}}^{N}\right)_{0 \leq i \leq N}=\left(x_{i}\right)_{0 \leq i \leq N}$, there are two cases:

1) if one of the simulated points is outside $D$, we are finished, $Z^{N}=0$;

2 ) if not, one of the processes $\left(X_{t}^{N}\right)_{t_{i} \leq t \leq t_{i+1}}$ may have left $D$ even if $X_{t_{i}}^{N} \in D$ and $X_{t_{i+1}}^{N} \in D$. The $N$ processes $\left(\left(X_{t}^{N}\right)_{t_{i} \leq t \leq t_{i+1}}\right)_{0 \leq i \leq N-1}$ conditioned by $\left(X_{t_{i}}^{N}=x_{i}\right)_{0 \leq i \leq N}$ are independent and of Brownian bridge type: thus, the simulation of the non exit on each interval is reduced to draw $N$ extra independent Bernoulli variables, whose parameters are

$$
\mathbb{P}\left(\forall t \in\left[t_{i}, t_{i+1}\right] X_{t}^{N} \in D \mid X_{t_{i}}^{N}=x_{i}, X_{t_{i+1}}^{N}=x_{i+1}\right):=p\left(x_{i}, x_{i+1}, T / N\right),
$$

the probability of non exit of some Brownian bridge.

Hence, the feasibility of the algorithm reduces to our ability to compute explicitly $p\left(x_{i}, x_{i+1}, T / N\right)$.

If $D=\left\{y \in \mathbb{R}^{d}: n .(y-z)>0\right\}$ is a half-space, this can be done (see e.g. [18]), and one has

$$
p\left(x_{i}, x_{i+1}, T / N\right)=1-\exp \left(-2 \frac{\left[n \cdot\left(x_{i}-z\right)\right]\left[n \cdot\left(x_{i+1}-z\right)\right]}{n \cdot\left(\left[\sigma \sigma^{*}\left(x_{i}\right)\right] n\right)(T / N)}\right)
$$

for $x_{i} \in D$ and $x_{i+1} \in D$ (and for non degenerate matrix $\sigma \sigma^{*}$ in the normal direction at $\partial D$ ).

For more general domains, there is no explicit formula for $p\left(x_{i}, x_{i+1}, \epsilon\right)$. Nevertheless, one can compute asymptotic expansions of this probability w.r.t. $\epsilon$, using large deviation arguments (see [1,2]). Unfortunately, each term in this expansion (even the first one) depends on the geometry of the boundary and are in general complicated to evaluate numerically (an example is considered in Sect. 2.3). Moreover, one does not know how many terms are needed to ensure that the global error is of order $N^{-1}$ for example.

Our approach described below consists in locally approximating the domain by an appropriate half-space, so that the procedure described above can be fully implemented (in particular, using formula such as (11)). Moreover, Theorem 2.3 states that the associated weak error is of order $N^{-1}$, the best that we could hope.

We now turn to the description of the algorithm. 


\subsection{Algorithm and analysis of the error}

We first define a set of half-spaces which will be usefull in the following:

Definition 2.1. For $x$ close to $\partial D$, i.e. $x \in V_{\partial D}(R)$ (where $R$ is the constant involved in Proposition (1.1) taking $\gamma=n$ ), we denote by $D(x)$ the half-space delimited by the tangent hyper-plane to $\partial D$ at $z=\pi_{\partial D}^{n}(x)$ :

$$
D(x):=\left\{y \in \mathbb{R}^{d}: n(z) \cdot(y-z)>0\right\} .
$$

We now describe how to approximately simulate $\mathbf{1}_{T<\tau} f\left(X_{T}\right)$ by some quantity $Z^{N}$. The simulation of the scheme follows an iterative routine, with $N$ steps for the discretization times. To begin, we set $X_{0}^{N}=x \in D$.

Assume that $X_{t_{i}}^{N}$ has been simulated, that $X_{t_{i}}^{N} \in D$ (otherwise, the simulation can be stopped and $Z^{N}=0$ ), and that until $t_{i}$, no exit has been detected (the associated rule is described below).

We now proceed to the simulation on the interval $\left[t_{i}, t_{i+1}\right]$. One keeps using the continuous Euler scheme (9) given by $X_{t}^{N}=X_{t_{i}}^{N}+B\left(X_{t_{i}}^{N}\right)\left(t-t_{i}\right)+\sigma\left(X_{t_{i}}^{N}\right)\left(W_{t}-W_{t_{i}}\right)$, but the domain $D$ is fictively modified on each interval.

Once $X_{t_{i+1}}^{N}$ is simulated, different situations may happen.

Case 1. If $X_{t_{i+1}}^{N} \notin D$, the process has clearly left $D$, and $Z^{N}=0$.

Case 2. If $X_{t_{i+1}}^{N} \in D$, an exit may have occurred in $\left[t_{i}, t_{i+1}\right]$.

Case 2.a. $X_{t_{i}}^{N}$ is far from $\partial D: X_{t_{i}}^{N} \notin V_{\partial D}(R)$.

One decides that there has been no exit, since the probability that $\left(X_{t}^{N}\right)_{t_{i} \leq t \leq t_{i+1}}$ covers a fixed distance $R>0$ during a short period $T / N$, is very small.

Case 2.b. $X_{t_{i}}^{N}$ is close to $\partial D: X_{t_{i}}^{N} \in V_{\partial D}(R)$.

Here, one simulates the exit of $\left(X_{t}^{N}\right)_{t_{i} \leq t \leq t_{i+1}}$ from the half-space $D\left(X_{t_{i}}^{N}\right)$ (see Def. 2.1), which approximates $D$ in a neighborhood of $X_{t_{i}}^{N}: D\left(X_{t_{i}}^{N}\right):=\left\{y \in \mathbb{R}^{d}: n\left(z_{i}\right) \cdot\left(y-z_{i}\right)>0\right\}$ with $z_{i}=\pi_{\partial D}^{n}\left(X_{t_{i}}^{N}\right)$. Now, draw an extra independent Bernoulli random variable $U_{i}$ with parameter (see formula (11))

$$
1-\exp \left(-2 \frac{\left[n\left(z_{i}\right) \cdot\left(X_{t_{i}}^{N}-z_{i}\right)\right]\left[n\left(z_{i}\right) \cdot\left(X_{t_{i+1}}^{N}-z_{i}\right)\right]}{n\left(z_{i}\right) \cdot\left(\left[\sigma \sigma^{*}\left(X_{t_{i}}^{N}\right)\right] n\left(z_{i}\right)\right)(T / N)}\right) .
$$

If $U_{i}=0$, there is an exit, and $Z^{N}=0$. Otherwise, one continues the iteration.

At the end of $N$-th step, if no exit has been detected, one puts $Z^{N}=f\left(X_{T}^{N}\right)$, or $Z^{N}=0$ in the opposite case. We define

$$
\tau^{N}=\inf \left\{t_{i}>0: X_{t_{i}}^{N} \notin D\right\} \wedge \inf \left\{t>0: X_{\varphi(t)}^{N} \in V_{\partial D}(R) \text { and } X_{t}^{N} \notin D\left(X_{\varphi(t)}^{N}\right)\right\} .
$$

Then, one has $Z^{N}=\mathbf{1}_{T<\tau^{N}} f\left(X_{T}^{N}\right)$.

Remark 2.2. To get a lower variance for the simulations, instead of simulating $Z^{N}$, one should use $\mathbb{E}\left(Z^{N} \mid X_{0}^{N}, \cdots, X_{t_{i}}^{N}, \cdots, X_{T}^{N}\right)$, which is equal to $f\left(X_{T}^{N}\right) \prod_{i=0}^{N-1} p\left(X_{t_{i}}^{N}, X_{t_{i+1}}^{N}\right)$, where

$$
p\left(x_{i}, x_{i+1}\right)=\left\{\begin{array}{cl}
0 & \text { if } x_{i} \text { or } x_{i+1} \notin D \\
1 & \text { if } x_{i} \in D, x_{i+1} \in D \text { and } d\left(x_{i}, \partial D\right) \geq R \\
1-\exp \left(-2 \frac{\left[n\left(z_{i}\right) \cdot\left(x_{i}-z_{i}\right)\right]\left[n\left(z_{i}\right) \cdot\left(x_{i+1}-z_{i}\right)\right]}{n\left(z_{i}\right) \cdot\left(\left[\sigma \sigma^{*}\left(x_{i}\right)\right] n\left(z_{i}\right)\right)(T / N)}\right) & \text { if } x_{i} \in D, x_{i+1} \in D \\
& \text { and } d\left(x_{i}, \partial D\right) \leq R, \text { with } z_{i}=\pi_{\partial D}^{n}\left(x_{i}\right) .
\end{array}\right.
$$

Before stating the results on the rate of convergence of this algorithm, we specify some appropriate assumptions on the smoothness of the coefficients and the non degeneracy of the diffusion coefficient. 
Assumption (S). $B($.$) is a C_{b}^{5}\left(\mathbb{R}^{d}, \mathbb{R}^{d}\right)$ function and $\sigma($.$) is a C_{b}^{5}\left(\mathbb{R}^{d}, \mathbb{R}^{d} \otimes \mathbb{R}^{d}\right)$ function.

Assumption (E). The matrix $\sigma \sigma^{*}$ is uniformly elliptic: for some $\sigma_{0}>0$, one has

$$
\forall x \in \mathbb{R}^{d} \sigma \sigma^{*}(x) \geq \sigma_{0}^{2} I_{\mathbb{R}^{d} \otimes \mathbb{R}^{d}} .
$$

We analyze the error induced by this algorithm, for two types of hypotheses on the function $f$.

Hypothesis (H1). $f$ is a bounded measurable function, satisfying $d(\operatorname{supp}(\mathrm{f}), \partial \mathrm{D}) \geq \epsilon>0$.

Hypothesis (H2). $f$ is a $C_{b}^{5}(\bar{D}, \mathbb{R})$ function, satisfying the following condition of vanishing on $\partial D$ : $\forall z \in$ $\partial D \quad f(z)=L f(z)=L^{2} f(z)=0$.

The main result of this section is the following:

Theorem 2.3. Assume $(\mathbf{D}),(\mathbf{S})$ and $(\mathbf{E})$. Then, one has:

$$
\mathbb{E}_{x}\left[Z^{N}-\mathbf{1}_{T<\tau} f\left(X_{T}\right)\right]=O\left(N^{-1}\right),
$$

provided that $f$ satisfies $(\mathbf{H 1})$ or $(\mathbf{H 2})$.

\subsection{Proofs}

Denote by $\mathcal{L}_{z}$, the operator on $C^{2}$ functions defined by

$$
\mathcal{L}_{z} u(x)=\sum_{i=1}^{d} B_{i}(z) \partial_{i} u(x)+\frac{1}{2} \sum_{i=1}^{d} \sum_{j=1}^{d}\left(\sigma \sigma^{*}(z)\right)_{i, j} \partial_{i, j}^{2} u(x)
$$

(for $t \in\left[t_{i}, t_{i+1}\right), \mathcal{L}_{X_{t_{i}}^{N}}$ plays the role of the infinitesimal generator of $X_{t}^{N}$ ).

If we set $\hat{\tau}^{N}=\tau^{N} \wedge \inf \left\{t>0: X_{t}^{N} \notin D(R)\right\}$ and $\hat{Z}^{N}=\mathbf{1}_{T<\hat{\tau}^{N}} f\left(X_{T}^{N}\right)$, then $\mathbb{E}_{x}\left|\hat{Z}^{N}-Z^{N}\right|=O_{\text {pol }}(N)$. Indeed, one has

$$
\mathbb{P}_{x}\left(T \wedge \tau^{N} \neq T \wedge \hat{\tau}^{N}\right) \leq K(T) \exp \left(-c \frac{R^{2}}{T / N}\right),
$$

estimate which can be easily derived from classical upper bounds for large deviation probability (see e.g. Lem. 4.1 from [12]): if $\mathrm{d} U_{t}=b_{t} \mathrm{~d} t+a_{t} \mathrm{~d} W_{t}$ defines an Ito process with uniformly bounded coefficients, one has

$$
\forall \eta \geq 0 \quad \mathbb{P}\left(\sup _{t \in\left[S, S^{\prime}\right]}\left\|U_{t}-U_{S}\right\| \geq \eta\right) \leq K(T) \exp \left(-c \frac{\eta^{2}}{\Delta}\right),
$$

for $S$ and $S^{\prime}$ two stopping times, bounded by $T$, such that $0 \leq S^{\prime}-S \leq \Delta$.

So, it remains to prove that $\mathbb{E}_{x}\left[\hat{Z}^{N}-\mathbf{1}_{T<\tau} f\left(X_{T}\right)\right]=O\left(N^{-1}\right)$.

Following [12], let introduce the PDE solved by $\mathbb{E}_{x}\left[\mathbf{1}_{T<\tau} f\left(X_{T}\right)\right]$. For $(t, x) \in[0, T] \times \mathbb{R}^{d}$, we set

$$
v(t, x):=\mathbb{E}_{x}\left[\mathbf{1}_{T-t<\tau} f\left(X_{T-t}\right)\right] .
$$

The function $v(t, x)$ is at least of class $C^{2,4}([0, T) \times \bar{D}, \mathbb{R}) \cap C^{0}\left([0, T) \times \mathbb{R}^{d}, \mathbb{R}\right)$ and satisfies a parabolic PDE of second order with Cauchy and Dirichlet conditions, i.e.

$$
\left\{\begin{array}{cl}
\partial_{t} v+L v=0 & \text { for }(t, x) \in[0, T) \times \bar{D} \\
v(t, x)=0 & \text { for }(t, x) \in[0, T] \times D^{c} \\
v(T, x)=f(x) & \text { for } x \in D
\end{array}\right.
$$


where $L$ is defined in (3). Actually, the smoothness of $v$ follows from that of the transition density of $\left(X_{t}\right)_{t \geq 0}$ (also called the fundamental solution of (17), see [17] Th. 16.3). Then, one has:

$$
\begin{aligned}
\mathbb{E}\left[\hat{Z}^{N}-\mathbf{1}_{T<\tau} f\left(X_{T}\right)\right] & =\mathbb{E}_{x}\left[\mathbf{1}_{T<\hat{\tau}^{N}} v\left(T, X_{T}^{N}\right)\right]-v(0, x) \\
& =\mathbb{E}_{x}\left[v\left(T \wedge \hat{\tau}^{N}, X_{T \wedge \hat{\tau}^{N}}^{N}\right)-v\left(0, X_{0}^{N}\right)\right]-\mathbb{E}_{x}\left[\mathbf{1}_{T \geq \hat{\tau}^{N}} v\left(\hat{\tau}^{N}, X_{\hat{\tau}^{N}}^{N}\right)\right]
\end{aligned}
$$

The second term of the r.h.s of (18) is of order $N^{-1}$, applying the following lemma, whose proof is postponed in Section 2.2.3.

Lemma 2.4. Assume (D), (S) and (E). Then, provided that $f$ satisfies $(\mathbf{H 1})$ or $(\mathbf{H 2})$, one has:

$$
\mathbb{E}_{x}\left[\mathbf{1}_{T \geq \hat{\tau}^{N}} v\left(\hat{\tau}^{N}, X_{\hat{\tau}^{N}}^{N}\right)\right]=O\left(N^{-1}\right) .
$$

It remains to prove that the first term in the r.h.s. of (18) has the expected order. For this, we shall apply Ito's formula to develop the above expression. To avoid some problems with the estimates on the derivatives of $v$, we first consider the case of smooth functions $f$.

\subsubsection{First case: $f$ satisfies $\mathbf{( H 2 )}$}

Under this hypothesis, derivatives of $v$ are uniformly bounded on $[0, T] \times \bar{D}$ : there is a function $K(T)$, such that for all multi-index $\alpha$ of length $|\alpha| \leq 4$, we have

$$
\forall(t, x) \in[0, T] \times \bar{D} \quad\left|\partial_{x}^{\alpha} v(t, x)\right| \leq K(T) .
$$

This is immediately derived from classical results for linear equations of parabolic type: we refer to [17] Theorem 5.2. (p. 320), for fuller statement. In fact, less regularity conditions on $B$ and $\sigma$ than required in (S) are needed for this result: the additional smoothness is used for Malliavin calculus computations when $f$ satisfies (H1) (see Lem. 2.7).

The application of Ito's formula in the first term in the r.h.s. of (18) is actually not straightforward since the derivatives of the function $v$ have discontinuities on the boundary $\partial D$. To overcome this difficulty, let us consider the orthogonal projection on $\bar{D}$ of $X_{t \wedge \hat{\tau}^{N}}^{N}$ (see Assertion iv) of Proposition 1.1): one knows from Proposition 3.1 from [12] that this is a continuous semimartingale with decomposition

$$
\mathrm{d}\left(\pi_{D}^{n}\left(X_{t \wedge \hat{\tau}^{N}}^{N}\right)\right)=\mathbf{1}_{t<\hat{\tau}^{N}}\left(\mathbf{1}_{X_{t}^{N} \in D} \mathrm{~d} X_{t}^{N}+\mathbf{1}_{X_{t}^{N} \notin D} \mathrm{~d} X_{t}^{N, \partial D}+\frac{1}{2} n\left(X_{t}^{N}\right) \mathrm{d} L_{t}^{0}\left(F^{n}\left(X_{.}^{N}\right)\right)\right) .
$$

$L_{t}^{0}(Y)$ is the 1-dimensional local time of the continuous semimartingale $Y$ at time $t$ and level 0 . The process $\left(X_{t}^{N, \partial D}\right)_{0 \leq t \leq T}$ is obtained from $\left(X_{t}^{N}\right)_{0 \leq t \leq T}$ by smooth transformations, which need not to be explicited here: the only thing important is that this remains an Ito process, with bounded coefficients. 
Since $v$ vanishes outside $D$, one has $v\left(T \wedge \hat{\tau}^{N}, X_{T \wedge \hat{\tau}^{N}}^{N}\right)=v\left(T \wedge \hat{\tau}^{N}, \pi_{D}^{n}\left(X_{T \wedge \hat{\tau}^{N}}^{N}\right)\right)$ and $\partial_{t} v(t, x)=0$ for $x \notin D$. Hence, one gets, using $\partial_{t}+L v=0$ in $D$,

$$
\begin{aligned}
\mathbb{E}_{x}\left[v\left(T \wedge \hat{\tau}^{N}, X_{T \wedge \hat{\tau}^{N}}^{N}\right)-v\left(0, X_{0}^{N}\right)\right]= & \mathbb{E}_{x}\left[\left.\int_{0}^{T \wedge \hat{\tau}^{N}} \mathrm{~d} t \mathbf{1}_{X_{t}^{N} \in D}\left(\mathcal{L}_{z} v+\partial_{t} v\right)\right|_{z=X_{\varphi(t)}^{N}}\left(t, X_{t}^{N}\right)\right] \\
& +\mathbb{E}_{x}\left[\int_{0}^{T \wedge \hat{\tau}^{N}} \frac{1}{2} \sum_{l=1}^{d} \partial_{l} v\left(t, X_{t}^{N}\right) n_{l}\left(X_{t}^{N}\right) \mathrm{d} L_{t}^{0}\left(F^{n}\left(X_{.}^{N}\right)\right)\right] \\
& +\mathbb{E}_{x}\left[\int_{0}^{T \wedge \hat{\tau}^{N}} \sum_{l=1}^{d} \partial_{l} v\left(t, \pi_{D}^{n}\left(X_{t}^{N}\right)\right) \mathbf{1}_{X_{t}^{N} \notin D} \mathrm{~d} X_{l, t}^{N, \partial D}\right. \\
& \left.+\frac{1}{2} \sum_{l=1}^{d} \sum_{m=1}^{d} \partial_{l, m}^{2} v\left(t, \pi_{D}^{n}\left(X_{t}^{N}\right)\right) \mathbf{1}_{X_{t}^{N} \notin D} d\left\langle X_{l, .}^{N, \partial D}, X_{m, .}^{N, \partial D}\right\rangle_{t}\right] .
\end{aligned}
$$

Let denote by $\bar{v}$ a function of class $C^{2,4}\left([0, T] \times \mathbb{R}^{d}, \mathbb{R}\right)$ which coincides with $v$ on $[0, T] \times \bar{D}$ : this extended function can be chosen such that its derivatives of type (19) are uniformly bounded on $[0, T] \times \mathbb{R}^{d}$ (see e.g. Sect. IV.4 in [17]). With this notation, one gets

$$
\begin{aligned}
\mathbb{E}_{x}\left[v\left(T \wedge \hat{\tau}^{N}, X_{T \wedge \hat{\tau}^{N}}^{N}\right)-v\left(0, X_{0}^{N}\right)\right]= & \mathbb{E}_{x}\left[\left.\int_{0}^{T} \mathrm{~d} t \mathbf{1}_{t<\hat{\tau}^{N}}\left(\mathcal{L}_{z} \bar{v}-L \bar{v}\right)\right|_{z=X_{\varphi(t)}^{N}}\left(t, X_{t}^{N}\right)\right] \\
& +\mathbb{E}_{x}\left[\int_{0}^{T \wedge \hat{\tau}^{N}} u_{1}(t) \mathrm{d} L_{t}^{0}\left(F^{n}\left(X_{.}^{N}\right)\right)\right]+\mathbb{E}_{x}\left[\int_{0}^{T \wedge \hat{\tau}^{N}} u_{2}(t) \mathbf{1}_{X_{t}^{N} \notin D} \mathrm{~d} t\right] \\
:= & C_{1}(N)+C_{2}(N)+C_{3}(N),
\end{aligned}
$$

where the adapted processes $u_{1}$ and $u_{2}$ are uniformly bounded. We now prove that terms $C_{1}(N), C_{2}(N)$ and $C_{3}(N)$ are of order $N^{-1}$.

\section{1) Term $C_{1}(N)$}

Using $\mathbf{1}_{t<\hat{\tau}^{N}}=\mathbf{1}_{\varphi(t)<\hat{\tau}^{N}}-\mathbf{1}_{\varphi(t)<\hat{\tau}^{N} \leq t}$, we obtain

$$
\begin{aligned}
C_{1}(N) & =\int_{0}^{T} \mathrm{~d} t \mathbb{E}_{x}\left[\left.\mathbf{1}_{\varphi(t)<\hat{\tau}^{N}}\left(\mathcal{L}_{z} \bar{v}-L \bar{v}\right)\right|_{z=X_{\varphi(t)}^{N}}\left(t, X_{t}^{N}\right)\right]-\int_{0}^{T} \mathrm{~d} t \mathbb{E}_{x}\left[\left.\mathbf{1}_{\varphi(t)<\hat{\tau}^{N} \leq t}\left(\mathcal{L}_{z} \bar{v}-L \bar{v}\right)\right|_{z=X_{\varphi(t)}^{N}}\left(t, X_{t}^{N}\right)\right] \\
& :=C_{4}(N)-C_{5}(N) .
\end{aligned}
$$

It is easy to see that $\left.\left(\mathcal{L}_{z} \bar{v}-L \bar{v}\right)\right|_{z=X_{\varphi(t)}^{N}}\left(t, X_{t}^{N}\right)$ has the form $\sum_{1 \leq|\alpha| \leq 2} c_{\alpha} \partial_{x}^{\alpha} \bar{v}\left(t, X_{t}^{N}\right)\left[g_{\alpha}\left(X_{t}^{N}\right)-g_{\alpha}\left(X_{\varphi(t)}^{N}\right)\right]$, with $g_{\alpha}=B_{i}$ or $\left(\sigma \sigma^{*}\right)_{i, j}$. Hence, Ito's formula in term $C_{4}(N)$ yields easily that $C_{4}(N)=O\left(N^{-1}\right)$. For the second term, one gets

$$
\left|C_{5}(N)\right| \leq K(T) \mathbb{E}_{x}\left[\int_{0}^{T} \mathrm{~d} t \mathbf{1}_{\varphi(t)<\hat{\tau}^{N} \leq t} \max _{0 \leq i \leq N-1} \sup _{u \in\left[t_{i}, t_{i+1}\right]}\left\|X_{u}^{N}-X_{t_{i}}^{N}\right\|_{\mathbb{R}^{d}}\right]=O\left(N^{-1}\right),
$$

where we used the obvious inequality $\int_{0}^{T} \mathrm{~d} t \mathbf{1}_{\varphi(t)<\hat{\tau}^{N} \leq t} \leq T N^{-1}$ and some standard uniform $L_{p^{-}}$estimates on $\sup _{t}\left\|X_{t}^{N}\right\|_{\mathbb{R}^{d}}$. Some refinements would even show that $\mathbb{E}_{x}\left(\max _{0 \leq i \leq N-1} \sup _{u \in\left[t_{i}, t_{i+1}\right]}\left\|X_{u}^{N}-X_{t_{i}}^{N}\right\|_{\mathbb{R}^{d}}\right)=$ $O\left(N^{-1 / 2} \sqrt{\log N}\right)$, and thus $C_{5}(N)$ is actually negligible compared to $N^{-1}$. 
This proves that $C_{1}(N)$ has the expected order.

2) Terms $C_{2}(N)$ and $C_{3}(N)$.

Since the local time is a non-negative measure and the processes $u_{1}, u_{2}$ are bounded, one obtains that $\left|C_{2}(N)\right|+\left|C_{3}(N)\right| \leq K(T) \mathbb{E}_{x}\left[L_{T \wedge \hat{\tau}^{N}}^{0}\left(F^{n}\left(X_{.}^{N}\right)\right)+\int_{0}^{T} \mathbf{1}_{t<\hat{\tau}^{N}} \mathbf{1}_{X_{t}^{N} \notin D} \mathrm{~d} t\right]$. To complete the proof of Theorem 2.3 when $f$ satisfies (H2), apply the two following crucial technical lemmas: their proofs are given in Section 2.2.3.

Lemma 2.5. Assume (D), (S) and $(\mathbf{E})$. There is a function $K(T)$, such that

$$
\int_{0}^{T} \mathrm{~d} t \mathbb{P}_{x}\left(t<\hat{\tau}^{N}, X_{t}^{N} \notin D\right) \leq K(T) N^{-3 / 2} .
$$

Lemma 2.6. Assume $(\mathbf{D}),(\mathbf{S})$ and $(\mathbf{E})$. There is a function $K(T)$, such that

$$
\mathbb{E}_{x}\left[L_{T \wedge \hat{\tau}^{N}}^{0}\left(F^{n}\left(X_{.}^{N}\right)\right)\right] \leq K(T) N^{-1}
$$

\subsubsection{Second case: $f$ satisfies (H1)}

The computations led in the previous case of smooth function $f$ can be performed in the same way, but the main difference now is that derivatives of $v(t, x)$ for $t$ close to $T$ may explode for irregular functions $f$.

To extend the validity of the above calculus led for $v$ with bounded derivatives to the current situation, one readily remarks that it is sufficient to apply the following result which gives all the needed estimates.

Lemma 2.7. Assume (D), (S), (E) and that $f$ satisfies $(\mathbf{H 1})$. Then, for all multi-index $\alpha$ with length $|\alpha| \leq 4$, for all $g \in C_{b}^{|\alpha|}\left(\mathbb{R}^{d}, \mathbb{R}\right)$, there is a function $K(T)$ (depending on $\|g\|_{C_{b}^{|\alpha|}}$ ), such that

$$
\begin{aligned}
\forall(s, x) \in[0, T) \times V_{\partial D}(\epsilon / 2) & \left|\partial_{x}^{\alpha} v(s, x)\right| & \leq K(T) \frac{\|f\|_{\infty}}{1 \wedge \epsilon^{|\alpha|}} \\
\forall(s, t) \in[0, T) \times[0, T] & \left|\mathbb{E}_{x}\left[\mathbf{1}_{s<\hat{\tau}^{N}} g\left(X_{t}^{N}\right) \partial_{x}^{\alpha} v\left(s, X_{s}^{N}\right)\right]\right| & \leq \frac{\|f\|_{\infty}}{1 \wedge \epsilon^{|\alpha|}} \frac{K(T)}{T^{\frac{|\alpha|}{2}}}
\end{aligned}
$$

Proof. The technical issue raised has been already overcome by the author in [12] (see Lem. 3.1), and the involved techniques can apply here in the same way. For this reason, we only recall the main arguments, and we refer to the cited paper for details.

First, the support condition on $f$ ensures that derivatives of $v$ are uniformly bounded near $\partial D$ : this is the first inequality of the above lemma. To derive the second inequality, we used Malliavin calculus techniques to rewrite the expectation using an integration by parts formula, following [3]. Because of the non smoothness of the killing time, it seems to be especially difficult: here again, the boundedness of derivatives of $v$ on $V_{\partial D}(\epsilon / 2)$ requires the use of Malliavin Calculus only for the law of $X^{N}$ restricted to the interior of $D$. But strictly inside $D$ (in fact, in $D \backslash V_{\partial D}(\epsilon / 2)$ ), there is no killing, so that a standard integration by parts formula can apply under (E) (up to some localizations in time and space). These arguments are adapted from Cattiaux [5,6].

\subsubsection{Proof of Lemmas 2.4-2.6}

The results are going to be progressively proved all together: since it is a bit intricate, we divide the proofs in several steps.

The technical difficulty is that one knows very few things about the law of the process $\left(X_{t}^{N}\right)_{0 \leq t \leq T}$ killed at time $\hat{\tau}^{N}$. The techniques used may be summed up in the following way:

1) to get local estimates, we use that on each interval, $\left(X_{t}^{N}\right)_{0 \leq t \leq T}$ is merely a Brownian motion;

2 ) to get global estimates, we use some occupation times formula, combined with accurate estimates of local times. 


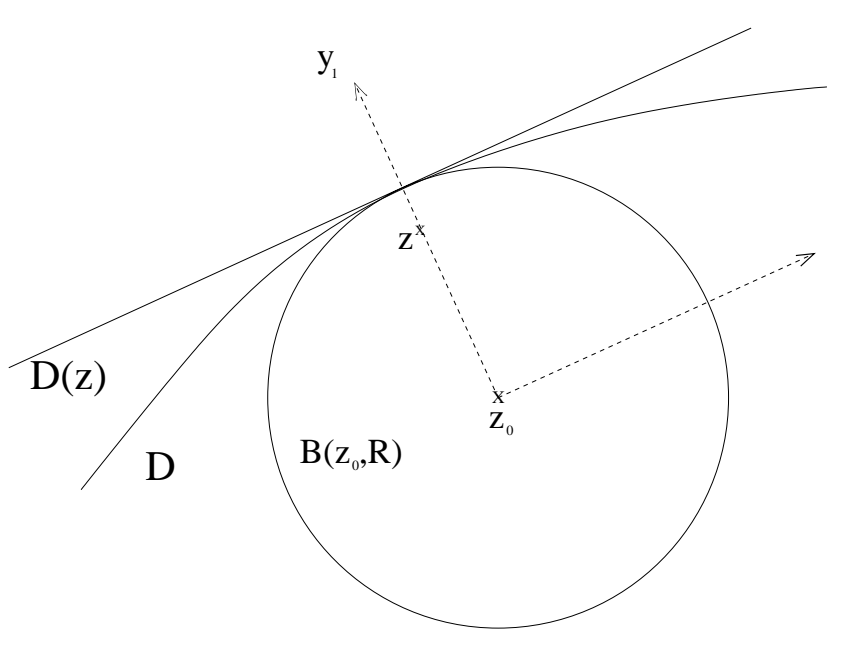

Figure 1. $D$ satisfies to the uniform interior sphere condition.

Step 1. Transformation of $\mathbb{P}_{x}\left(t<\hat{\tau}^{N}, X_{t}^{N} \notin D\right)$

Set $t \in(0, T]$, such that $t_{i}<t \leq t_{i+1}$. Using estimates (15), one obtains

$$
\begin{aligned}
\mathbb{P}_{x}\left(t<\hat{\tau}^{N}, X_{t}^{N} \notin D\right) & =\mathbb{P}_{x}\left(t<\hat{\tau}^{N}, X_{t}^{N} \notin D, X_{t_{i}}^{N} \in V_{\partial D}(R / 2)\right)+O_{\mathrm{pol}}(N) \\
& \leq \mathbb{E}_{x}\left(\mathbf{1}_{t_{i}<\hat{\tau}^{N}, X_{t_{i}}^{N} \in V_{\partial D}(R / 2)} \mathbb{P}\left(X_{t}^{N} \in D\left(X_{t_{i}}^{N}\right), X_{t}^{N} \notin D \mid \mathcal{F}_{t_{i}}\right)\right)+O_{\mathrm{pol}}(N) .
\end{aligned}
$$

We first prove that for $u \in(0, T / N]$ and $z \in D \cap V_{\partial D}(R / 2)$, one has

$$
\mathbb{P}_{z}\left(X_{u}^{N} \in D(z), X_{u}^{N} \notin D\right) \leq K(T) \sqrt{u} \exp \left(-c \frac{d^{2}(z, \partial D)}{u}\right),
$$

for some $c>0$. Since $D$ satisfies the uniform interior sphere condition and $d(z, \partial D) \leq R / 2 \leq R$, there exists $z_{0} \in D$ at distance $R$ from $\partial D$, such that the domain $D$ contains the ball $B\left(z_{0}, R\right)$ with center $z_{0}$ and radius $R$, tangent to $\partial D$ at point $\pi_{D}^{n}(z)$ (see Fig. 1): hence

$$
\mathbb{P}_{z}\left(X_{u}^{N} \in D(z), X_{u}^{N} \notin D\right) \leq \mathbb{P}_{z}\left(X_{u}^{N} \in D(z), X_{u}^{N} \notin B\left(z_{0}, R\right)\right)=\int_{D(z) \cap B^{c}\left(z_{0}, R\right)} p_{u}^{N}(z, y) \mathrm{d} y,
$$

where $p_{u}^{N}(z, y)$ is the Gaussian density of $X_{u}^{N} \mid X_{0}^{N}=z$, which satisfies $p_{u}^{N}(z, y) \leq \frac{K(T)}{u^{d / 2}} \exp \left(-c \frac{\|z-y\|^{2}}{u}\right)$. Up to a rotation of axes and to taking $z_{0}$ as new origin, we can consider w.l.o.g. that $D(z)=\left\{y \in \mathbb{R}^{d}: y_{1}<R\right\}$ and $B^{c}\left(z_{0}, R\right)=\left\{y \in \mathbb{R}^{d}:\|y\| \geq R\right\}$. Thus, the probability to evaluate can be upper bounded by

$$
\begin{aligned}
& \int_{y_{1}<R,\|y\| \geq R} \frac{K(T)}{u^{d / 2}} \exp \left(-c \frac{\left(R-d(z, \partial D)-y_{1}\right)^{2}+\sum_{i=2}^{d} y_{i}^{2}}{u}\right) \mathrm{d} y \\
\leq & \int_{0}^{R} \mathrm{~d} y_{1} \frac{K(T)}{\sqrt{u}} \exp \left(-c \frac{\left(R-d(z, \partial D)-y_{1}\right)^{2}}{u}\right) \int_{\sum_{i=2}^{d} y_{i}^{2} \geq R^{2}-y_{1}^{2}} \frac{\mathrm{d} y_{2} \cdots \mathrm{d} y_{d}}{u^{(d-1) / 2}} \exp \left(-c \frac{\sum_{i=2}^{\mathrm{d}} y_{i}^{2}}{u}\right)+O_{\mathrm{pol}}(N)
\end{aligned}
$$

by restricting the integral w.r.t. $y_{1}$ to the interval $[0, R]$ (the remainder term having an exponentially small contribution). The integral w.r.t. $y_{2}, \cdots, y_{d}$ can be easily simplified using the spheric coordinates in $\mathbb{R}^{d-1}$, and 
one obtains

$$
\begin{aligned}
\int_{\sum_{i=2}^{d} y_{i}^{2} \geq R^{2}-y_{1}^{2}} \frac{\mathrm{d} y_{2} \cdots \mathrm{d} y_{d}}{u^{(d-1) / 2}} \exp \left(-c \frac{\sum_{i=2}^{d} y_{i}^{2}}{u}\right) & =\int_{r^{2} \geq R^{2}-y_{1}^{2}} \frac{C r^{d-2}}{u^{(d-1) / 2}} \exp \left(-c \frac{r^{2}}{u}\right) \mathrm{d} r \\
& \leq C^{\prime} \exp \left(-c^{\prime} \frac{\left(R^{2}-y_{1}^{2}\right)}{u}\right) .
\end{aligned}
$$

Thus, one gets from (24), for new constants $K(T)$ and $c>0$,

$$
\begin{aligned}
& \mathbb{P}_{z}\left(X_{u}^{N} \in D(z), X_{u}^{N} \notin D\right) \leq \int_{0}^{R} \mathrm{~d} y_{1} \frac{K(T)}{\sqrt{u}} \exp \left(-c \frac{\left(R-d(z, \partial D)-y_{1}\right)^{2}+R^{2}-y_{1}^{2}}{u}\right)+O_{\mathrm{pol}}(N) \\
& =\frac{K(T)}{\sqrt{u}} \exp \left(-c \frac{(R-d(z, \partial D))^{2}+R^{2}}{u}\right) \int_{0}^{R} \mathrm{~d} y_{1} \exp \left(y_{1} \frac{2 c(R-d(z, \partial D))}{u}\right)+O_{\mathrm{pol}}(N) \\
& \leq \frac{K(T)}{\sqrt{u}} \exp \left(-c \frac{(R-d(z, \partial D))^{2}+R^{2}}{u}\right) \frac{\exp \left(R \frac{2 c(R-d(z, \partial D))}{u}\right)}{\frac{2 c(R-d(z, \partial D))}{u}}+O_{\mathrm{pol}}(N) \\
& \leq K(T) \sqrt{u} \exp \left(-c \frac{d^{2}(z, \partial D)}{u}\right)+O_{\mathrm{pol}}(N)
\end{aligned}
$$

using that $R-d(z, \partial D) \geq R / 2$ to bound from below the denominator at the last inequality. This proves the estimate (23). Combining this upper bound with (22), we obtain

$$
\mathbb{P}_{x}\left(t<\hat{\tau}^{N}, X_{t}^{N} \notin D\right) \leq K(T) \sqrt{\frac{T}{N}} \mathbb{E}_{x}\left(\mathbf{1}_{t_{i}<\hat{\tau}^{N}, X_{t_{i}}^{N} \in V_{\partial D}(R / 2)} \exp \left(-c \frac{d^{2}\left(X_{t_{i}}^{N}, \partial D\right)}{t-t_{i}}\right)\right)+O_{\mathrm{pol}}(N) .
$$

Step 2. Preliminary upper bound for $\mathbb{P}_{x}\left(t<\hat{\tau}^{N}, X_{t}^{N} \notin D\right)$

Obviously, one has

$$
\mathbb{P}_{x}\left(t<\hat{\tau}^{N}, X_{t}^{N} \notin D\right) \leq K(T) \sqrt{\frac{T}{N}} \mathbb{E}_{x}\left(\exp \left(-c \frac{d^{2}\left(X_{t_{i}}^{N}, \partial D\right)}{t-t_{i}}\right)\right)+O_{\mathrm{pol}}(N)
$$

If $p_{t}^{N}(x, y)$ denotes the density of $X_{t}^{N} \mid X_{0}^{N}=x$, it is well known that

$$
p_{t}^{N}(x, y) \leq \frac{K(T)}{t^{d / 2}} \exp \left(-c \frac{\|x-y\|^{2}}{t}\right)
$$

for any $(t, x, y) \in(0, T] \times \mathbb{R}^{d} \times \mathbb{R}^{d}$ (see Kusuoka and Stroock [16]), for some $c>0$. Then, apply the following lemma related to some kind of convolution of Gaussian kernels, to get

$$
\begin{aligned}
\mathbb{P}_{x}\left(t<\hat{\tau}^{N}, X_{t}^{N} \notin D\right) & \leq \frac{K(T)}{N} \frac{1}{\sqrt{t}}, \\
\int_{0}^{T} \mathbb{P}_{x}\left(t<\hat{\tau}^{N}, X_{t}^{N} \notin D\right) \mathrm{d} t & \leq \frac{K(T)}{N} .
\end{aligned}
$$

Estimate (27) will be improved later. 
Lemma 2.8. Assume (D). Put $c_{1}, c_{2}>0$. Then, for any $\delta, t \in(0, T]$, any $x \in \mathbb{R}^{d}$, any $k \geq 1$, one has

$$
\int_{\mathbb{R}^{d}} \mathrm{~d} z \frac{1}{t^{d / 2}} \exp \left(-c_{1} \frac{\|x-z\|^{2}}{t}\right) \exp \left(-c_{2} \frac{d^{2}(z, \partial D)}{\delta}\right) \leq K(T) \sqrt{\frac{\delta}{t+\delta}} \exp \left(-c_{1} \wedge c_{2} \frac{d^{2}(x, \partial D)}{\delta+t}\right)+O\left(\delta^{k}\right),
$$

for some constant $K(T)$ uniform in $x, t, \delta$, and $O\left(\delta^{k}\right)$ uniform in $x$ and $t$.

This lemma is easy to prove. If $D$ is a half-space, the estimate is straightforward to be obtained, simply using the convolution of Gaussian kernels. For a more general domain, one first needs to use local diffeomorphisms to reduce to the previous case. We do not give further details and refer to [11] for a complete proof.

Step 3. Transformation of $\mathbb{E}_{x}\left(\left(F^{n}\right)^{-}\left(X_{T \wedge \hat{\tau}^{N}}^{N}\right)\right)$

To study $\mathbb{E}_{x}\left[L_{T \wedge \hat{\tau}^{N}}^{0}\left(F^{n}\left(X^{N}\right)\right)\right]$, we prefer to consider $\mathbb{E}_{x}\left(\left(F^{n}\right)^{-}\left(X_{T \wedge \hat{\tau}^{N}}^{N}\right)\right)$, which is somehow equivalent. Combining the Markov property at time $t_{i}$ and the estimate (15), one has

$$
\begin{aligned}
& \mathbb{E}_{x}\left(\left(F^{n}\right)^{-}\left(X_{T \wedge \hat{\tau}^{N}}^{N}\right)\right)=\sum_{i=0}^{N-1} \mathbb{E}_{x}\left(\mathbf{1}_{t_{i}<\hat{\tau}^{N} \leq t_{i+1}}\left(F^{n}\right)^{-}\left(X_{\hat{\tau}^{N}}^{N}\right)\right) \\
= & \sum_{i=0}^{N-1} \mathbb{E}_{x}\left(\mathbf{1}_{t_{i}<\hat{\tau}^{N}} \mathbf{1}_{X_{t_{i}}^{N} \in V_{\partial D}(R)} \mathbb{E}_{X_{t_{i}}^{N}}\left(\mathbf{1}_{\hat{\tau}^{N} \leq T / N}\left(F^{n}\right)^{-}\left(X_{\hat{\tau}^{N}}^{N}\right)\right)\right)+O_{\text {pol }}(N) .
\end{aligned}
$$

Put $y \in D \cap V_{\partial D}(R)$ and let us introduce $\bar{\tau}^{N}=\left\{t>0: X_{t}^{N} \notin D(y)\right\}$. Applying once again (15), one gets

$$
\mathbb{E}_{y}\left(\mathbf{1}_{\hat{\tau}^{N} \leq T / N}\left(F^{n}\right)^{-}\left(X_{\hat{\tau}^{N}}^{N}\right)\right)=\mathbb{E}_{y}\left(\mathbf{1}_{\bar{\tau}^{N} \leq T / N}\left(F^{n}\right)^{-}\left(X_{\bar{\tau}^{N}}^{N}\right)\right)+O_{\mathrm{pol}}(N)
$$

Now, note that, since $F^{n}($.$) vanishes on \partial D$, one gets $\nabla F^{n}(x) \| n(x)$ for any $x \in \partial D$. Thus, combining second order Taylor's expansion formula around $\pi_{\partial D}^{n}(y)$ with the fact that $\left(X_{\bar{\tau}^{N}}^{N}-\pi_{\partial D}^{n}(y)\right) \perp n\left(\pi_{\partial D}^{n}(y)\right)$, one easily obtains $\left(F^{n}\right)^{-}\left(X_{\bar{\tau}^{N}}^{N}\right)=O\left(\left\|X_{\bar{\tau}}^{N}-\pi_{\partial D}^{n}(y)\right\|^{2}\right)$, and thus

$$
\mathbb{E}_{y}\left(\mathbf{1}_{\hat{\tau}^{N} \leq T / N}\left(F^{n}\right)^{-}\left(X_{\hat{\tau}^{N}}^{N}\right)\right) \leq C \mathbb{E}_{y}\left[\mathbf{1}_{\bar{\tau}^{N} \leq T / N}\left\|X_{\bar{\tau}}^{N}-\pi_{\partial D}^{n}(y)\right\|^{2}\right]+O_{\text {pol }}(N) .
$$

Up to rotating the axis, there is no restriction to assume that $n\left(\pi_{\partial D}^{n}(y)\right)$ lies along the first axis: $D(y)=\{z \in$ $\left.\mathbb{R}^{d}: z_{1}>y_{1}-d(y, \partial D)\right\}$. In that context, $\left(X_{t}^{N}\right)_{0 \leq t \leq T / N}$ is equal in law to

$$
X_{t}^{N}=y+B(y) t+\left(\begin{array}{c}
\Sigma_{1,1}(y) W_{1, t} \\
\sum_{j=1}^{d} \Sigma_{2, j}(y) W_{j, t} \\
\cdots \\
\sum_{j=1}^{d} \Sigma_{d, j}(y) W_{d, t}
\end{array}\right)
$$

where the inferior triangular matrix $\Sigma$ is defined by $\Sigma \Sigma^{*}=\sigma \sigma^{*}$. Since $\bar{\tau}^{N}=\inf \left\{t>0: X_{1, t}^{N} \notin D(y)\right\}=\inf \{t>$ $\left.0: B_{1}(y) t+\Sigma_{1,1}(y) W_{1, t}=-d(y, \partial D)\right\}$ and $\pi_{\partial D}^{n}(y)=\left(y_{1}-d(y, \partial D), y_{2}, \cdots, y_{d}\right)^{*}$, one obtains:

$$
\begin{aligned}
\left\|X_{\bar{\tau}^{N}}^{N}-\pi_{\partial D}^{n}(y)\right\|^{2} & =\sum_{i=2}^{d}\left(X_{i, \bar{\tau}^{N}}^{N}-y_{i}\right)^{2}=\sum_{i=2}^{d}\left(B_{i}(y) \bar{\tau}^{N}+\sum_{j=1}^{d} \Sigma_{i, j}(y) W_{j, \bar{\tau}^{N}}\right)^{2} \\
& \leq C\left(\left(\bar{\tau}^{N}\right)^{2}+d^{2}(y, \partial D)+\sum_{j=2}^{d} W_{j, \bar{\tau}}^{2}\right), \mathbb{E}_{y}\left[\mathbf{1}_{\bar{\tau}^{N} \leq T / N}\left\|X_{\bar{\tau}^{N}}^{N}-\pi_{\partial D}^{n}(y)\right\|^{2} \mid\left(W_{1, t}\right)_{0 \leq t \leq T / N}\right] \\
& \leq C \mathbf{1}_{\bar{\tau}^{N} \leq T / N}\left(\left(\bar{\tau}^{N}\right)^{2}+d^{2}(y, \partial D)+(d-1) \bar{\tau}^{N}\right) .
\end{aligned}
$$


Hence, we deduce that

$$
\begin{aligned}
\mathbb{E}_{y}\left[\mathbf{1}_{\bar{\tau}^{N} \leq T / N}\left\|X_{\bar{\tau}^{N}}^{N}-\pi_{\partial D}^{n}(y)\right\|^{2}\right] & \leq K(T) \mathbb{P}_{y}\left[\bar{\tau}^{N} \leq T / N\right]\left(T / N+d^{2}(y, \partial D)\right) \\
& \leq K(T) \exp \left(-c \frac{d^{2}(y, \partial D)}{T / N}\right)\left(T / N+d^{2}(y, \partial D)\right) \\
& \leq K(T) \frac{T}{N} \exp \left(-c \frac{d^{2}(y, \partial D}{T / N}\right)
\end{aligned}
$$

where we used the estimate (15) for the second inequality. Combining the above estimate with (28, 29), one gets:

$$
\mathbb{E}_{x}\left(\left(F^{n}\right)^{-}\left(X_{T \wedge \hat{\tau}^{N}}^{N}\right)\right) \leq K(T) \frac{T}{N} \sum_{i=0}^{N-1} \mathbb{E}_{x}\left(\mathbf{1}_{t_{i}<\hat{\tau}^{N}} \mathbf{1}_{X_{t_{i}}^{N} \in V_{\partial D}(R)} \exp \left(-c \frac{d^{2}\left(X_{t_{i}}^{N}, \partial D\right)}{T / N}\right)\right)+O_{\mathrm{pol}}(N)
$$

Step 4. Preliminary upper bound for $\mathbb{E}_{x}\left(\left(F^{n}\right)^{-}\left(X_{T \wedge \hat{\tau}^{N}}^{N}\right)\right)$

Using the same arguments as for (25) and (26), one easily obtains

$$
\mathbb{E}_{x}\left(\left(F^{n}\right)^{-}\left(X_{T \wedge \hat{\tau}^{N}}^{N}\right)\right) \leq K(T) \frac{T}{N}\left(1+\sum_{i=1}^{N-1} K(T) \sqrt{\frac{T / N}{t_{i}+T / N}}\right) \leq K(T) \sqrt{\frac{T}{N}}
$$

The above estimate is intermediary and is now going to be improved.

Step 5. Final upper bound for $\mathbb{E}_{x}\left[L_{T \wedge \hat{\tau}^{N}}^{0}\left(F^{n}\left(X^{N}\right)\right)\right]$ and $\mathbb{E}_{x}\left(\left(F^{n}\right)^{-}\left(X_{T \wedge \hat{\tau}^{N}}^{N}\right)\right)$

We first remark that for $i \geq 1$ and for any $t \in\left[t_{i-1}, t_{i}\right]$, one has

$$
\mathbb{E}_{x}\left(\mathbf{1}_{t_{i}<\hat{\tau}^{N}} \exp \left(-c \frac{d^{2}\left(X_{t_{i}}^{N}, \partial D\right)}{T / N}\right)\right) \leq K(T) \mathbb{E}_{x}\left(\mathbf{1}_{t<\hat{\tau}^{N}} \exp \left(-c^{\prime} \frac{d^{2}\left(X_{t}^{N}, \partial D\right)}{T / N}\right)\right)+O_{\mathrm{pol}}(N)
$$

for some constant $c^{\prime}>0$ and function $K(T)$ uniform in $t$ and $N$. Indeed, using $\mathbf{1}_{t_{i}<\hat{\tau}^{N}} \leq \mathbf{1}_{t<\hat{\tau}^{N}}$, one writes $\mathbb{E}_{x}\left(\mathbf{1}_{t_{i}<\hat{\tau}^{N}} \exp \left(-c \frac{d^{2}\left(X_{t_{i}}^{N}, \partial D\right)}{T / N}\right)\right) \leq \mathbb{E}_{x}\left(\mathbf{1}_{t<\hat{\tau}^{N}} \mathbb{E}\left(\exp \left(-c \frac{d^{2}\left(X_{t_{i}}^{N}, \partial D\right)}{T / N}\right) \mid \mathcal{F}_{t}\right)\right)$, and the result follows by combining a Gaussian upper bound for the density of $X_{t_{i}}^{N}$ conditionally on $\mathcal{F}_{t}$, with Lemma 2.8. Then, we easily deduce from (30) that

$$
\mathbb{E}_{x}\left(\left(F^{n}\right)^{-}\left(X_{T \wedge \hat{\tau}^{N}}^{N}\right)\right) \leq \frac{K(T)}{N}+K(T) \int_{0}^{T} \mathrm{~d} t \mathbb{E}_{x}\left(\mathbf{1}_{t<\hat{\tau}^{N}} \mathbf{1}_{X_{t}^{N} \in V_{\partial D}(R)} \exp \left(-c \frac{d^{2}\left(X_{t}^{N}, \partial D\right)}{T / N}\right)\right)
$$

To continue the analysis of the integral in r.h.s. of the above expression, we use the occupation times formula (see e.g. [28]). For this, remark that for $X_{t}^{N} \in V_{\partial D}(R)$, one has, using Proposition 1.1,
a) $d^{2}\left(X_{t}^{N}, \partial D\right)=\left(F^{n}\right)^{2}\left(X_{t}^{N}\right)$;
b) $\mathbf{1}_{X_{t}^{N} \in V_{\partial D}(R)}=\mathbf{1}_{\left|F^{n}\left(X_{t}^{N}\right)\right| \leq R}$;
c) $d<F^{n}\left(X_{.}^{N}\right), F^{n}\left(X_{.}^{N}\right)>=\left\|\sigma\left(X_{\varphi(t)}^{N}\right) n\left(\pi_{\partial D}^{n}\left(X_{t}^{N}\right)\right)\right\| \mathrm{d} t \geq \sigma_{0}^{2} \mathrm{~d} t$.

Hence, one gets

$$
\int_{0}^{T} \mathrm{~d} t \mathbb{E}_{x}\left(\mathbf{1}_{t<\hat{\tau}^{N}} \mathbf{1}_{X_{t}^{N} \in V_{\partial D}(R)} \exp \left(-c \frac{d^{2}\left(X_{t}^{N}, \partial D\right)}{T / N}\right)\right) \leq \frac{1}{\sigma_{0}^{2}} \int_{-R}^{R} \mathrm{~d} y \exp \left(-c \frac{y^{2}}{T / N}\right) \mathbb{E}_{x}\left[L_{T \wedge \hat{\tau}^{N}}^{y}\left(F^{n}\left(X^{N}\right)\right)\right] .
$$


To control the above integral, we prove that

$$
\mathbb{E}_{x}\left[L_{T \wedge \hat{\tau}^{N}}^{y}\left(F^{n}\left(X_{.}^{N}\right)\right)\right] \leq K(T)\left(|y|+N^{-1}\right) .
$$

Indeed, from Tanaka's formula (see [28]), it follows that

$$
\mathbb{E}_{x}\left[L_{T \wedge \hat{\tau}^{N}}^{y}\left(F^{n}\left(X_{.}^{N}\right)\right)\right]=2 \mathbb{E}_{x}\left[\left(F^{n}\left(X_{T \wedge \hat{\tau}^{N}}^{N}\right)-y\right)^{-}-\left(F^{n}(x)-y\right)^{-}+\int_{0}^{T} \mathbf{1}_{F^{n}\left(X_{t}^{N}\right) \leq y} \mathbf{1}_{t<\hat{\tau}^{N}} \mathrm{~d}\left(F^{n}\left(X_{t}^{N}\right)\right)\right] .
$$

On one hand, the triangular inequality combined with the preliminary estimate (31) yields

$$
\mathbb{E}_{x}\left|\left(F^{n}\left(X_{T \wedge \hat{\tau}^{N}}^{N}\right)-y\right)^{-}-\left(F^{n}(x)-y\right)^{-}\right| \leq \mathbb{E}_{x}\left|\left(F^{n}\left(X_{T \wedge \hat{\tau}^{N}}^{N}\right)\right)^{-}-\left(F^{n}(x)\right)^{-}\right|+2|y| \leq K(T) N^{-1 / 2}+2|y| .
$$

On the other hand, one clearly has $\mathbb{E}_{x}\left[\int_{0}^{T} \mathbf{1}_{F^{n}\left(X_{t}^{N}\right) \leq y} \mathbf{1}_{t<\hat{\tau}^{N}} \mathrm{~d}\left(F^{n}\left(X_{t}^{N}\right)\right)\right] \leq C \mathbb{E}_{x}\left[\int_{0}^{T} \mathbf{1}_{F^{n}\left(X_{t}^{N}\right) \leq y} \mathbf{1}_{t<\hat{\tau}^{N}} \mathrm{~d} t\right]$. Then, one distinguishes two cases:

1) If $y \leq 0$, one has $\mathbb{E}_{x}\left[\int_{0}^{T} \mathbf{1}_{F^{n}\left(X_{t}^{N}\right) \leq y} \mathbf{1}_{t<\hat{\tau}^{N}} \mathrm{~d} t\right] \leq K(T) N^{-1}$ using that $\mathbf{1}_{F^{n}\left(X_{t}^{N}\right) \leq y} \leq \mathbf{1}_{F^{n}\left(X_{t}^{N}\right) \leq 0}=$ $\mathbf{1}_{X_{t}^{N} \notin D}$ combined with $(27)$;

2) If $y>0$, then $\mathbb{E}_{x}\left[\int_{0}^{T} \mathbf{1}_{F^{n}\left(X_{t}^{N}\right) \leq y} \mathbf{1}_{t<\hat{\tau}^{N}} \mathrm{~d} t\right]=\mathbb{E}_{x}\left[\int_{0}^{T} \mathbf{1}_{F^{n}\left(X_{t}^{N}\right) \leq 0} \mathbf{1}_{t<\hat{\tau}^{N}} \mathrm{~d} t\right]+\mathbb{E}_{x}\left[\int_{0}^{T} \mathbf{1}_{0<F^{n}\left(X_{t}^{N}\right) \leq y}\right.$ $\left.\mathbf{1}_{t<\hat{\tau}^{N}} \mathrm{~d} t\right]$. The first term of the r.h.s. is bounded by $K(T) N^{-1}$ as before. For the second, one may once again apply occupation times formula, as for $(33)$, to get $\int_{0}^{T} \mathrm{~d} t \mathbb{E}_{x}\left(1_{0<F^{n}\left(X_{t}^{N}\right) \leq y} \mathbf{1}_{t<\hat{\tau}^{N}}\right) \leq$ $\frac{1}{\sigma_{0}^{2}} \int_{0}^{y} \mathrm{~d} z \mathbb{E}_{x}\left[L_{T \wedge \hat{\tau}^{N}}^{z}\left(F^{n}\left(X_{.}^{N}\right)\right)\right] \leq K(T) y$, simply using $\sup _{|y| \leq R} \mathbb{E}_{x}\left[L_{T \wedge \hat{\tau}^{N}}^{y}\left(F^{n}\left(X_{.}^{N}\right)\right)\right] \leq K(T)$, which is a direct consequence of (35).

Hence, one has proved $\mathbb{E}_{x}\left[\int_{0}^{T} \mathbf{1}_{F^{n}\left(X_{t}^{N}\right) \leq y} \mathbf{1}_{t<\hat{\tau}^{N}} \mathrm{~d}\left(F^{n}\left(X_{t}^{N}\right)\right)\right] \leq K(T)\left(|y|+N^{-1}\right)$ : reminding (35), this shows that $\mathbb{E}_{x}\left[L_{T \wedge \hat{\tau}^{N}}^{y}\left(F^{n}\left(X^{N}\right)\right)\right] \leq K(T)\left(|y|+N^{-1 / 2}\right)$. Plugging this estimate in (33), and then in (32), one obtains

$$
\mathbb{E}_{x}\left(\left(F^{n}\right)^{-}\left(X_{T \wedge \hat{\tau}^{N}}^{N}\right)\right) \leq K(T) N^{-1} .
$$

To derive (34), restart computations from (35) using $K(T) N^{-1}$ instead of $K(T) N^{-1 / 2}$ for an upper bound for $\mathbb{E}_{x}\left(\left(F^{n}\right)^{-}\left(X_{T \wedge \hat{\tau}^{N}}^{N}\right)\right)$. Taking $y=0$ in $(34)$ proves Lemma 2.6.

Step 6. Final upper bound for $\int_{0}^{T} d t \mathbb{P}_{x}\left(t<\hat{\tau}^{N}, X_{t}^{N} \notin D\right)$

Using (25), one gets:

$$
\begin{aligned}
\int_{0}^{T} \mathrm{~d} t \mathbb{P}_{x}\left(t<\hat{\tau}^{N}, X_{t}^{N} \notin D\right) \leq & K(T)\left(\frac{T}{N}\right)^{3 / 2} \sum_{i=0}^{N-1} \mathbb{E}_{x}\left(\mathbf{1}_{t_{i}<\hat{\tau}^{N}, X_{t_{i}}^{N} \in V_{\partial D}(R / 2)} \exp \left(-c \frac{d^{2}\left(X_{t_{i}}^{N}, \partial D\right)}{T / N}\right)\right) \\
& +O_{\mathrm{pol}}(N) \leq K(T) N^{-3 / 2}
\end{aligned}
$$

where for the last inequality, we apply the same arguments from (30) to (36). This proves Lemma 2.5.

\section{Step 7. Proof of Lemma 2.4}

The key point is to note that $v(t,$.$) vanishes outside D$. Thus, the quantity $\mathbb{E}_{x}\left[\mathbf{1}_{T \geq \hat{\tau}^{N}} v\left(\hat{\tau}^{N}, X_{\hat{\tau}^{N}}^{N}\right)\right]$ can be estimated in the same way that we have proceeded for $\mathbb{E}_{x}\left(\left(F^{n}\right)^{-}\left(X_{T \wedge \hat{\tau}^{N}}^{N}\right)\right)$ in $(28)$ : this gives the same upper bound than in $(36)$, i.e. $O\left(N^{-1}\right)$. The proof is complete. 


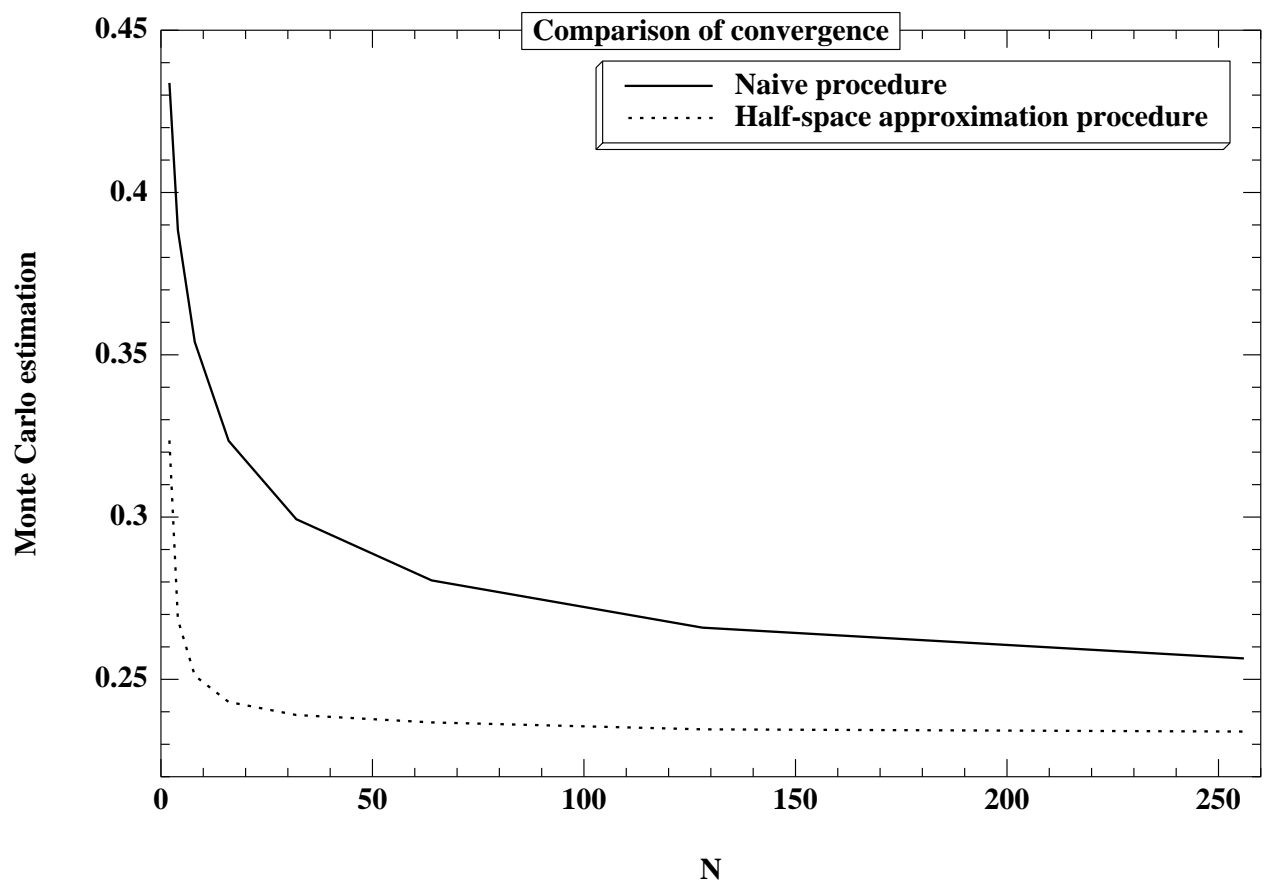

Figure 2. Comparison of convergence.

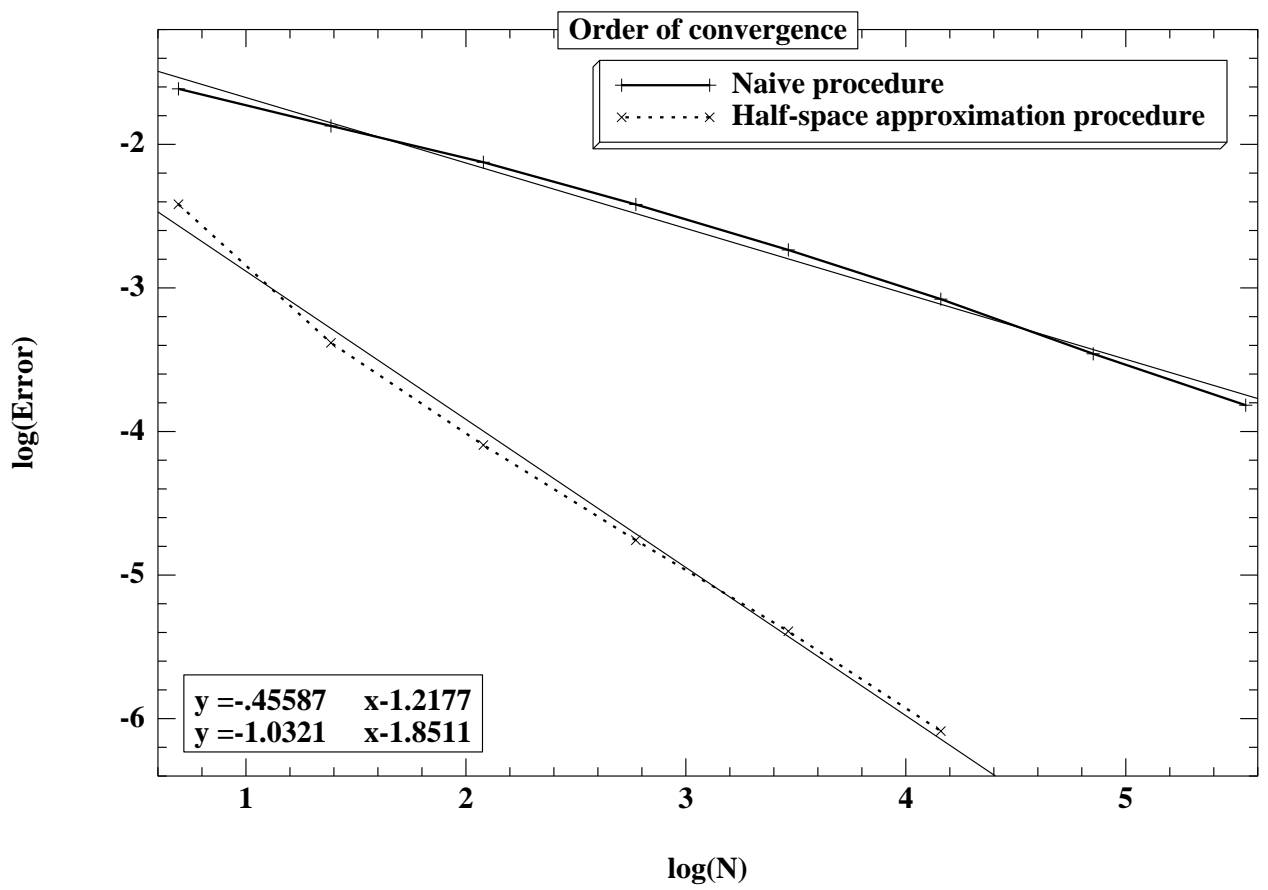

Figure 3. Order of convergence. 


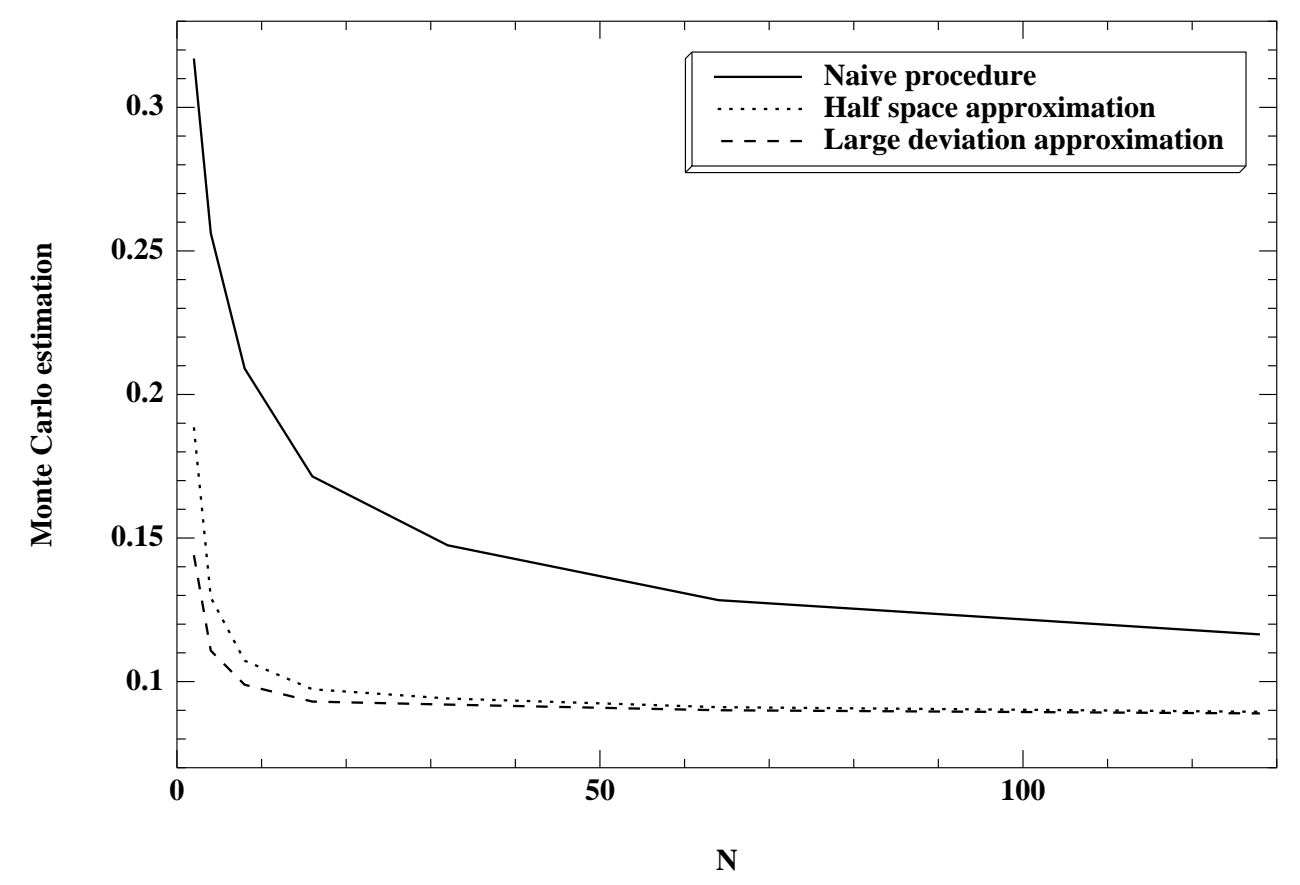

FiguRE 4. Comparison with large deviation estimates.

\subsection{Numerical experiments}

To test the accuracy of our procedure, we consider a 2-dimensional diffusion process $\left(X_{t}\right)_{0 \leq t \leq T}$ killed at its exit of the unit sphere: $D=\left\{x \in \mathbb{R}^{2}:\|x\|<1\right\}$. The coefficients which define $\left(X_{t}\right)_{0 \leq t \leq T}$ are the following ones:

$$
B(x)=\left(\begin{array}{c}
-x_{2} \\
x_{1}
\end{array}\right), \quad \sigma(x)=\left(\begin{array}{cc}
0 & \left(2+\sin \left(x_{1}+x_{2}\right)\right) / 3 \\
\left(2+\cos \left(x_{1}+x_{2}\right)\right) / 3 & 0
\end{array}\right) .
$$

We set $X_{0}=0$ and $T=1$. The function $f$ of interest is chosen as $f(x)=\left(\|x\|^{2}-1\right)^{2}$. The current and further computations have been performed on a PC computer (Pentium III processor, $800 \mathrm{MHz}$ ), using a code program in $C$. To remove the Monte-Carlo method error in order to get only the discretization error, we have taken large number of simulations (namely $M=1000000$ ), with various number of time steps $N$ (from $N=2$ to $N=256$ ). Figure 2 represents the quantities evaluated by thoses simulations using either the naive procedure (which involves only the simulation of a discrete time process), either the half-space approximation procedure from this paper. One observes that the improved procedure converges much quickly to the true value (which is unknown here).

We can also test the order of convergence, by ploting the errors w.r.t. $N$ in log scales, assuming the relation $|\operatorname{Error}(N)| \sim N^{-\alpha}$ : the opposite of the slope then gives approximatively the order of convergence $\alpha$. Here, the quantity of reference for the true value is taken to be the result of a Romberg extrapolation (see [32]) using half-space approximation algorithm with $N=256$ and $N=512$, for $M=5000000$ paths. For the naive procedure, the estimated order of convergence (see Fig. 3) is $\alpha=0.45587$ (the theoritical rate is $\alpha=0.5$ ), while for half-space approximation, one gets $\alpha=1.0321$, which confirms somehow Theorem 2.3.

Another interesting issue is to compare our procedure with the one based on sharp large deviation estimates to compute $p\left(x_{i}, x_{i+1}, \epsilon\right)$ defined by $(10)$ (see $\left.[1,2]\right)$. We consider here a 2-dimensional Brownian motion starting from 0 , killed at its exit from the unit sphere $D=\left\{x \in \mathbb{R}^{2}:\|x\|<1\right\}$, and we compute the probability of exit 
before time $T=1$ (i.e. $f \equiv 1$ ). At the first order (see [1]), one may simply consider

$$
p\left(x_{i}, x_{i+1}, \epsilon\right) \sim \exp \left(-\frac{u\left(x_{i}, x_{i+1}\right)}{2 \epsilon}\right)
$$

where $u\left(x_{i}, x_{i+1}\right)=\min _{\phi \in \partial D}\left(\left\|x_{i}-\phi\right\|+\left\|x_{i+1}-\phi\right\|\right)^{2}-\left\|x_{i}-x_{i+1}\right\|^{2}$.

Figure 4 gives the Monte-Carlo results w.r.t. the number of time steps $N$, for the naive procedure, the half-space approximation and the large deviation approximation, using $M=100000$ simulations. The true value is about 0.0878 . The large deviation approximation behaves slightly better than the procedure based on half-space approximation concerning the accuracy for a given number $N$ of time steps. However, if we focus on the computational time, the algorithm we propose is much quicker.

\begin{tabular}{|c|c|c|c|}
\hline & Naive procedure & Half-space approximation & Large deviation approximation \\
\hline \hline Estimated value & 0.147030 & 0.091367 & 0.091141 \\
\hline Relative error & $67 \%$ & $4.1 \%$ & $3.8 \%$ \\
\hline $\begin{array}{c}\text { 95\% confidence interval } \\
\text { on the estimated value }\end{array}$ & {$[0.149270,0.144790]$} & {$[0.093044,0.089690]$} & {$[0.092817,0.089465]$} \\
\hline Time & $2.40 \mathrm{~s}$ & $2.99 \mathrm{~s}$ & $31.17 \mathrm{~s}$ \\
\hline
\end{tabular}

The above tabular sums up some results obtained for $N=32$ time steps. It appears than the half-space approximation procedure and the large deviation one have got merely the same precision, but the first one is about 10 times quicker. This is due to the difficulty of computing $u\left(x_{i}, x_{i+1}\right)$ by finding the minimizing point $\phi \in \partial D$. This example illustrates also that the proposed procedure is almost as quick as the naive one, while being much more accurate.

\section{ReFlected Diffusions}

In this section, the process $\left(X_{t}\right)_{t \geq 0}$ is a diffusion process taking its values in some domain $D$, with an instantaneous oblique reflection on $\partial \bar{D}$ : it is defined by

$$
X_{t}=x+\int_{0}^{t} B\left(X_{s}\right) \mathrm{d} s+\int_{0}^{t} \sigma\left(X_{s}\right) \mathrm{d} W_{s}+\int_{0}^{t} \gamma\left(X_{s}\right) \mathrm{d} k_{s}
$$

where

- $\left(W_{t}\right)_{t \geq 0}$ is a $d$-dimensional Brownian motion;

- the so-called local time $k_{t}$ is a process increasing only on $\partial D: k_{t}=\int_{0}^{t} \mathbf{1}_{X_{t} \in \partial D} \mathrm{~d} k_{s}$.

The domain $D$ is supposed to satisfy assumption (D), while the unit inward vector field $\gamma$ is non tangent to $\partial D$ (assumption $(\Gamma)$ ).

Our objective is now to compute, using Monte-Carlo simulations, $\mathbb{E}_{x}\left[f\left(X_{T}\right)-\int_{0}^{T} h\left(X_{t}\right) \mathrm{d} k_{t}\right]$, for given measurable functions $f$ and $h$, and for a fixed time $T$.

Projected Euler scheme. One way to do consists in using a projected Euler scheme (see papers from Saisho [29]; Slominski [31]; Pettersson [26]; Constantini et al. [7] and references therein): this is the discrete version of the Skorohod problem. We present the algorithm, in its generalized version to the case of oblique reflection. If the discretization times are given by $t_{i}=i T / N$, the projected Euler scheme $\left(X_{t_{i}}^{N}\right)_{0 \leq i \leq N}$ and its 
associated local time are defined by:

$$
\left\{\begin{aligned}
X_{0}^{N} & =x \\
Y_{t_{i+1}}^{N} & =X_{t_{i}}^{N}+B\left(X_{t_{i}}^{N}\right) T / N+\sigma\left(X_{t_{i}}^{N}\right)\left(W_{t_{i+1}}-W_{t_{i}}\right) \\
X_{t_{i+1}}^{N} & =\pi_{D}^{\gamma}\left[Y_{t_{i+1}}^{N}\right] \\
k_{t_{i+1}}^{N} & =k_{t_{i}}^{N}+\left(F^{\gamma}\right)^{-}\left[Y_{t_{i+1}}^{N}\right] .
\end{aligned}\right.
$$

One may have some problems to define $\pi_{D}^{\gamma}$ and $F^{\gamma}$ if the simulation $Y_{t_{i+1}}^{N}$ is too far from $D$ (namely, $d\left(Y_{t_{i+1}}^{N}, \partial D\right)>$ $R$ ): anyway, this situation occurs with an exponentially small probability (w.r.t. $N$ ), and if so, one may redraw the simulation, this having no incidence on the accuracy of the algorithm.

Hence, to simulate $f\left(X_{T}\right)-\int_{0}^{T} h\left(X_{t}\right) \mathrm{d} k_{t}$, one may use

$$
Z^{N}:=f\left(X_{T}^{N}\right)-\sum_{i=0}^{N-1} h\left(\pi_{\partial D}^{\gamma}\left(X_{t_{i}}^{N}\right)\right)\left(k_{t_{i+1}}^{N}-k_{t_{i}}^{N}\right) .
$$

Constantini et al. [7] has performed an analysis of the rate of convergence of such an approximation, and has proved, under some assumptions, that

$$
\mathbb{E}_{x}\left(Z^{N}-\left\{f\left(X_{T}\right)-\int_{0}^{T} h\left(X_{t}\right) \mathrm{d} k_{t}\right\}\right)=O\left(N^{-1 / 2}\right) .
$$

This rate is achieved in some simple particular cases. Actually, the rate obtained in [7] is $N^{-1 / 2+\epsilon}$ for any $\epsilon>0$ in the case of normal reflection, but using some arguments from this paper, it is possible to obtain the rate $N^{-1 / 2}$, even for the case of oblique reflection.

Penalty method. Another approach has been developed by Menaldi [22], Lions and Sznitman [20], Liu [21], Pettersson [27], Kanagawa and Saisho [15] among others. It consists in replacing the local time term in (38) by a drift type term, which value is zero inside $D$ and rapidly grows w.r.t. the distance to $\partial D$, outside $D$. Derivation of some rates of convergence is available, but only for the strong approximation, and we refer the reader to the cited papers for further details.

An appropriate Markov chain approximation at random discretization times has been studied by Milshtein [23]. His procedure requires at each step near the boundary to change coordinates, and by the way, the algorithm seems to be difficult to implement.

Let us also mention a paper by Hausenblas [14], who uses excursions theory to build a numerical scheme in the particular case of half-space domain.

Exact simulation when $D$ is a half-space. Lépingle $[18,19]$ has pointed out that, when the coefficients $B$, $\sigma, \gamma$ are constants and when $D$ is a half-space, an exact simulation of $\left(X_{t}, k_{t}\right)$ is available.

To state a precise formulation, we define $D=\left\{z \in \mathbb{R}^{d}:(z-y) \cdot n>0\right\}$ and consider the solution of $X_{t}=$ $x+B t+\sigma W_{t}+\gamma k_{t}$, which is obliquely reflected in $D$. In that case, it is easy to check, from the statement of the Skorohod problem, that $k_{t}$ is explicitly given by:

$$
k_{t}=\frac{1}{n \cdot \gamma} \max \left(0, \sup _{0 \leq s \leq t}-\left(x+B s+\sigma W_{s}-y\right) \cdot n\right) .
$$


Thus, we can simulate $\left(X_{t}, k_{t}\right)$ owing the

Proposition 3.1. Set $a \in \mathbb{R}^{d}$ and $c \in \mathbb{R}$. If $U \stackrel{\text { law }}{=} \mathcal{N}\left(0, t \mathrm{I}_{d}\right)$ and $V \stackrel{\text { law }}{=} \mathcal{E}(1 / 2 t)$ independent of $U$, one has

$$
\left(W_{t}, \sup _{0 \leq s \leq t}\left(a \cdot W_{s}+c s\right)\right) \stackrel{\operatorname{law}}{=}\left(U, \frac{1}{2}\left[a \cdot U+c t+\sqrt{|a|^{2} V+(a \cdot U+c t)^{2}}\right]\right) .
$$

Then, Lépingle derives from this result a discretization scheme for non constant function $B$ and $\sigma$ when the domain is a half-space. He derives the rate of convergence, but only for the strong approximation.

Our approach. Our contribution to the problem of simulation of reflected diffusions is threefold.

1) We derive a fully implementable procedure for general reflected diffusions in smooth domains, by taking profit from the fact that one can simulate exactly a reflected diffusion with constant coefficients in a half-space.

2) We prove in Theorems 3.2 and 3.3, the associated weak error can be of order $N^{-1}$ instead of $N^{-1 / 2}$ in the previous works.

3) We allow the function $f$ to be only measurable provided that a support condition is fulfilled, whereas as far as we know, only smooth functions $f$ have been considered before (see [7]).

Theorem 3.2 below has been announced without proofs by the author in [13]: in the cited paper, the algorithm suggested is slightly different and less accurate than the one below, when one computes expectations of integral w.r.t. local time. We now turn to the description of the current algorithm.

\subsection{Algorithm and analysis of the error}

\subsubsection{Algorithm}

To begin, set $X_{0}^{N}=x$ and $k_{0}^{N}=0$. We assume that $X_{t_{i}}^{N} \in \bar{D}$ and $k_{t_{i}}^{N}$ are defined and we now construct $X_{t_{i+1}}^{N}$ and $k_{t_{i+1}}^{N}$.

Case a. $X_{t_{i}}^{N} \notin V_{\partial D}(R)$.

We can consider that there is no reflection between $t_{i}$ and $t_{i+1}$, which is false only with an exponentially small probability w.r.t. $N$. Thus, we set

$$
X_{t_{i+1}}^{N}=X_{t_{i}}^{N}+B\left(X_{t_{i}}^{N}\right)\left(t_{i+1}-t_{i}\right)+\sigma\left(X_{t_{i}}^{N}\right)\left(W_{t_{i+1}}-W_{t_{i}}\right) \quad \text { and } \quad k_{t_{i+1}}^{N}=k_{t_{i}}^{N} .
$$

If $X_{t_{i+1}}^{N} \notin D$, we reject the simulation and redraw a new one: anyhow, this occurs with a negligible probability.

Case b. $X_{t_{i}}^{N} \in V_{\partial D}(R)$.

Step 1. We set $s=\pi_{\partial D}^{\gamma}\left(X_{t_{i}}^{N}\right)$ for the projection of $X_{t_{i}}^{N}$ on $\partial D$ parallel to $\gamma$, and we define $D(s)=$ $\left\{z \in \mathbb{R}^{d}:(z-s) \cdot n(s)>0\right\}$ the half-space delimited by the tangent plane to $\partial D$, at $s$ (see Fig. 5).

Step 2. Let $\left(Y_{t}^{i}\right)_{t_{i} \leq t \leq t_{i+1}}$ be the diffusion with constant coefficients, reflected on $\partial D$ in the direction $\gamma(s)$ : this is defined by

$$
Y_{t}^{i}=X_{t_{i}}^{N}+B\left(X_{t_{i}}^{N}\right)\left(t-t_{i}\right)+\sigma\left(X_{t_{i}}^{N}\right)\left(W_{t}-W_{t_{i}}\right)+\gamma(s)\left(k_{t}^{N}-k_{t_{i}}^{N}\right)
$$

Note that $\left(Y_{t_{i+1}}^{i}, k_{t_{i+1}}^{N}-k_{t_{i}}^{N}\right)$ can be simulated using Proposition 3.1. On the rare event $\left\{Y_{t_{i+1}}^{i} \notin D(R)\right\}$, restart the above simulation.

Step 3. If $Y_{t_{i+1}}^{i} \in \bar{D}$ (this occurs most of the time), we set $X_{t_{i+1}}^{N}=Y_{t_{i+1}}^{i}$. Otherwise, if $Y_{t_{i+1}}^{i} \notin \bar{D}$, set $X_{t_{i+1}}^{N}=\pi_{D}^{\gamma}\left(Y_{t_{i+1}}^{i}\right)$ (see Figure 5). The quantity $\left(F^{\gamma}\right)^{-}\left(Y_{t_{i+1}}^{i}\right)$ also contributes to increase the local time between $t_{i}$ and $t_{i+1}$. 


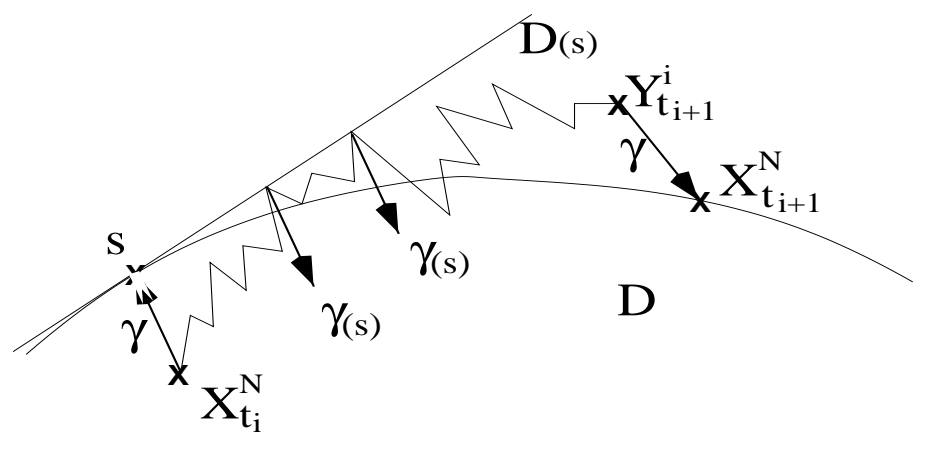

FIGURE 5. Description of the algorithm near the boundary.

We propose to approximate the quantity of interest $f\left(X_{T}\right)-\int_{0}^{T} h\left(X_{t}\right) \mathrm{d} k_{t}$ by

$$
Z^{N}:=f\left(X_{T}^{N}\right)-\sum_{i=0}^{N-1} \mathbf{1}_{X_{t_{i}}^{N} \in V_{\partial D}(R)} h\left(\pi_{\partial D}^{\gamma}\left(X_{t_{i}}^{N}\right)\right)\left(k_{t_{i+1}}^{N}-k_{t_{i}}^{N}+\left(F^{\gamma}\right)^{-}\left(Y_{t_{i+1}}^{i}\right)\right) .
$$

The quantity $Z^{N}$ is different from that suggested in [13], by the term $\left(F^{\gamma}\right)^{-}\left(Y_{t_{i+1}}^{i}\right)$, which turns to be important to get a rate of convergence equal to $N^{-1}$ when $h \neq 0$.

In the following theorems, we state that the associated weak error is of order $N^{-1}$ when the reflection direction $\gamma$ is so-called conormal, that is $\gamma \equiv \gamma^{c}$, where $\gamma^{c}$ is given in Definition 1.2. Actually, it is known that nice symmetry properties are available in that context (see e.g. $[24,25]$ ). In our analysis of the algorithm, similar properties will be used.

Some standard assumptions on the coefficients involved in the definition of $\left(X_{t}\right)_{0 \leq t \leq T}$ are needed. These are the following ones.

Assumption (S). B(.) is a $C_{b}^{5}\left(\bar{D}, \mathbb{R}^{d}\right)$ function and $\sigma($.$) is a C_{b}^{5}\left(\bar{D}, \mathbb{R}^{d} \otimes \mathbb{R}^{d}\right)$ function.

Assumption (E). The matrix $\sigma \sigma^{*}$ is uniformly elliptic: for some $\sigma_{0}>0$, one has

$$
\forall x \in \bar{D} \quad \sigma \sigma^{*}(x) \geq \sigma_{0}^{2} I_{\mathbb{R}^{d} \otimes \mathbb{R}^{d}} .
$$

\subsubsection{Main results}

We first consider the case $h \equiv 0$, i.e. we focus on the approximation of $\mathbb{E}_{x}\left(f\left(X_{T}\right)\right)$ : hence, $Z^{N}=f\left(X_{T}^{N}\right)$. As for the case of killed diffusions, two types of hypotheses on the function $f$ are considered.

Hypothesis (H1). $f$ is a bounded measurable function, satisfying $d(\operatorname{supp}(\mathrm{f}), \partial \mathrm{D}) \geq \epsilon>0$.

Hypothesis (H2). $f$ is a $C_{b}^{5}(\bar{D}, \mathbb{R})$ function, satisfying the following condition of vanishing on $\partial D$ : $\forall z \in$ $\partial D \nabla f \cdot \gamma(z)=\nabla(L f) \cdot \gamma(z)=0$.

Our main result is:

Theorem 3.2. Assume (D), (Г), (S), (E) and that $f$ satisfies (H1) or (H2). 
If the reflection is conormal, then

$$
\mathbb{E}_{x}\left[f\left(X_{T}^{N}\right)-f\left(X_{T}\right)\right]=O\left(N^{-1}\right)
$$

In the other cases, the error is of order $N^{-1 / 2}$.

If we allow $h$ to be different from 0 , considering the weak approximation of $f\left(X_{T}\right)-\int_{0}^{T} h\left(X_{t}\right) \mathrm{d} k_{t}$, we use $Z^{N}$ defined in (42). Since the case of measurable functions $f$ has been considered in Theorem 3.2, we assume here that $f$ and $h$ are both smooth functions, which satisfy the following compatibility conditions.

Hypothesis (H3). The function $f$ (resp. $h$ ) is a $C_{b}^{5}(\bar{D}, \mathbb{R})$ (resp. $C_{b}^{4}(\partial D, \mathbb{R})$ ) function, satisfying the following compatibility conditions on $\partial D: \forall z \in \partial D \quad \nabla f \cdot \gamma(z)-h(z)=\nabla(L f) \cdot \gamma(z)=0$.

Theorem 3.3. Assume (D), (Г), (S), (E) and that $f$ satisfies (H3).

If the reflection is conormal, then

$$
\mathbb{E}_{x}\left[Z^{N}-\left(f\left(X_{T}\right)-\int_{0}^{T} h\left(X_{t}\right) \mathrm{d} k_{t}\right)\right]=O\left(N^{-1}\right)
$$

In the other cases, the error is of order $N^{-1 / 2}$.

Thus, in the case of conormal reflection, Theorems 3.2 and 3.3 ensure that the algorithm converges faster than that using projected Euler scheme [7]. Furthermore, numerical experiments illustrate that this seems to be also true when the reflection is not conormal, even if no theoretical result confirms this numerical fact.

\subsection{Proofs}

For $(t, x) \in[0, T] \times \bar{D}$, we set

$$
v(t, x):=\mathbb{E}_{x}\left[f\left(X_{T-t}\right)-\int_{0}^{T-t} h\left(X_{s}\right) \mathrm{d} k_{s}\right]
$$

Here again, the approximation error is analyzed using the PDE solved by $v$.

Under Assumptions $(\mathbf{D}),(\Gamma),(\mathbf{S})$ and $(\mathbf{E})$, the function $v(t, x)$ is of class $C^{2,4}([0, T) \times \bar{D}, \mathbb{R})$ and satisfies a parabolic PDE of second order with Neumann condition, i.e.

$$
\left\{\begin{array}{cl}
\partial_{t} v+L v=0 & \text { for }(t, x) \in[0, T) \times \bar{D} \\
(\nabla v \cdot \gamma)(t, x)=h(x) & \text { for }(t, x) \in[0, T) \times \partial D \\
v(T, x)=f(x) & \text { for } x \in D
\end{array}\right.
$$

the operator $L$ being given by (3) (we have assumed that $f$ is a bounded measurable function and $h$ is smooth, say of class $C^{3}$ ). In the following, it might be useful to rewrite expectations of integral w.r.t. the local time $k_{t}$ in terms of surface integral involving the transition density $p_{t}(x, y)$ of the Markov process $\left(X_{t}\right)_{t \geq 0}$. Namely, one has:

$$
\mathbb{E}_{x}\left[\int_{0}^{T} h\left(t, X_{t}\right) \mathrm{d} k_{t}\right]=\frac{1}{2} \int_{0}^{T} \mathrm{~d} t \int_{\partial D} \mathrm{~d} s a^{\gamma}(s) p_{t}(x, s) h(t, s),
$$


where $a^{\gamma}$ is defined in (5). Indeed, let $u(t, x)=\mathbb{E}_{x}\left[-\int_{0}^{T-t} h\left(s, X_{s}\right) \mathrm{d} k_{s}\right]$ be the solution of (44) with $f \equiv 0$ as Cauchy condition and $h(t, x)$ as Neumann condition. Then, one has:

$$
\mathbb{E}_{x}\left(\int_{0}^{T} h\left(t, X_{t}\right) \mathrm{d} k_{t}\right)=\mathbb{E}_{x}\left(u\left(T, X_{T}\right)-u(0, x)\right)=\int_{0}^{T} \mathrm{~d} t \int_{D} \mathrm{~d} z\left(\partial_{t} p_{t}(x, z) u(t, z)+p_{t}(x, z) \partial_{t} u(t, z)\right)
$$

For fixed $x$, the function $(t, z) \mapsto p_{t}(x, z)$ satisfies the Kolmogorov forward equation $\partial_{t} p_{t}(x, z)=L^{*} p_{t}(x, z)$ with the boundary condition $-\frac{1}{2} a^{\gamma^{*}}(s) \nabla p_{t}(x, s) \cdot \gamma^{*}(s)+b(s) p_{t}(x, s)=0$ for any $s \in \partial D$, where $a^{\gamma^{*}}$ and $b$ are given in formula (6). Hence, the cited formula yields

$$
\int_{D} \mathrm{~d} z \partial_{t} p_{t}(x, z) u(t, z)=\int_{D} \mathrm{~d} z p_{t}(x, z) L u(t, z)+\frac{1}{2} \int_{\partial D} a^{\gamma}(s) p_{t}(x, s) \nabla u(t, s) \cdot \gamma(s) \mathrm{d} s,
$$

and using the PDE solved by $u$, one completes the proof of (45).

\subsubsection{Proof of Theorems 3.2 and 3.3 in the case of smooth functions $f$ and $h$}

We first derive a general decomposition of the error. Using $v(T,)=.f($.$) , one has$

$$
\mathbb{E}_{x}\left(f\left(X_{T}^{N}\right)-\sum_{i=0}^{N-1} \mathbf{1}_{X_{t_{i}}^{N} \in V_{\partial D}(R)} h\left(\pi_{\partial D}^{\gamma}\left(X_{t_{i}}^{N}\right)\right)\left(k_{t_{i+1}}^{N}-k_{t_{i}}^{N}+\left(F^{\gamma}\right)^{-}\left(Y_{t_{i+1}}^{i}\right)\right)\right)-v(0, x):=\sum_{i=0}^{N-1}\left(A_{i}+B_{i}\right)
$$

where $A_{i}=\mathbb{E}_{x}\left(\mathbf{1}_{X_{t_{i}}^{N} \notin V_{\partial D}(R)}\left[v\left(t_{i+1}, X_{t_{i+1}}^{N}\right)-v\left(t_{i}, X_{t_{i}}^{N}\right)\right]\right)$,

$$
B_{i}=\mathbb{E}_{x}\left(\mathbf{1}_{X_{t_{i}}^{N} \in V_{\partial D}(R)}\left[v\left(t_{i+1}, X_{t_{i+1}}^{N}\right)-v\left(t_{i}, X_{t_{i}}^{N}\right)-h\left(\pi_{\partial D}^{\gamma}\left(X_{t_{i}}^{N}\right)\right)\left(k_{t_{i+1}}^{N}-k_{t_{i}}^{N}+\left(F^{\gamma}\right)^{-}\left(Y_{t_{i+1}}^{i}\right)\right)\right]\right),
$$

where $Y_{t_{i+1}}^{i}$ is defined in Step 2. Under Assumption (H2) or (H3), the function $v$ is at least a $C^{2,4}([0, T] \times \bar{D}, \mathbb{R})$ function, with uniformly bounded derivatives (see Th. 5.3, p. 320 [17]): there is a function $K(T)$, such that for all multi-index $\alpha$ of length $|\alpha| \leq 4$, we have

$$
\forall(t, x) \in[0, T] \times \bar{D} \quad\left|\partial_{x}^{\alpha} v(t, x)\right| \leq K(T) .
$$

From now on, we consider a function defined on $[0, T) \times \mathbb{R}^{d}$, which coincides with $v$ on $[0, T) \times \bar{D}$, and which has the same regularity properties than those on $[0, T) \times \bar{D}$ (see Sect. IV.4 [17]): we still denote it by $v$.

We first focus on terms $A_{i}$. This is the easy part of the analysis since it is merely related to the behavior far from the boundary. For $t \in\left[t_{i}, t_{i+1}\right]$, define $Z_{t}=X_{t_{i}}^{N}+B\left(X_{t_{i}}^{N}\right)\left(t-t_{i}\right)+\sigma\left(X_{t_{i}}^{N}\right)\left(W_{t}-W_{t_{i}}\right)$. If one introduces $\tau^{N}=\inf \left\{t>t_{i}: d\left(Z_{t}, \partial D\right) \leq R / 2\right\}$, one remarks that on $\left\{\tau^{N}>t_{i+1}\right\}$, one has $X_{t_{i+1}}^{N}=Z_{t_{i+1}}$, referring to Case a) of the algorithm. Since $\mathbb{P}_{x}\left(\tau^{N} \leq t_{i+1}, X_{t_{i}}^{N} \notin V_{\partial D}(R)\right)=O_{\text {pol }}(N)$ (use estimate (15)), one readily obtains that

$$
A_{i}=\mathbb{E}_{x}\left(\mathbf{1}_{X_{t_{i}}^{N} \notin V_{\partial D}(R)}\left[v\left(t_{i+1}, Z_{t_{i+1}}\right)-v\left(t_{i}, X_{t_{i}}^{N}\right)\right]\right)+O_{\mathrm{pol}}(N)
$$

Using the notation $\mathcal{L}_{z}$ for the infinitesimal generator of $Z_{t}$ on $\left[t_{i}, t_{i+1}\right.$ ) (see (13)), two applications of Ito's formula, combined with $\left.\left(\mathcal{L}_{z} v+\partial_{t} v\right)\right|_{z=X_{t_{i}}^{N}}\left(t, Z_{t}\right)=0$ for $t=t_{i}$, easily yield:

$$
\begin{aligned}
A_{i} & =\mathbb{E}_{x}\left(\left.\mathbf{1}_{X_{t_{i}}^{N} \notin V_{\partial D}(R)} \int_{t_{i}}^{t_{i+1}} \mathrm{~d} t\left(\mathcal{L}_{z} v+\partial_{t} v\right)\right|_{z=X_{t_{i}}^{N}}\left(t, Z_{t}\right)\right)+O_{\mathrm{pol}}(N) \\
& =\mathbb{E}_{x}\left(\left.\mathbf{1}_{X_{t_{i}}^{N} \notin V_{\partial D}(R)} \int_{t_{i}}^{t_{i+1}} \mathrm{~d} t \int_{t_{i}}^{t} \mathrm{~d} r\left(\mathcal{L}_{z}+\partial_{t}\right)\left(\mathcal{L}_{z} v+\partial_{t} v\right)\right|_{z=X_{t_{i}}^{N}}\left(r, Z_{r}\right)\right)+O_{\mathrm{pol}}(N)
\end{aligned}
$$


This proves that $A_{i}=O\left(N^{-2}\right)$ uniformly in $i$. Hence, summing up this estimate in (46), it follows that these terms have a global contribution of order $N^{-1}$.

We now consider the estimation of terms $B_{i}$. This corresponds to the crucial contribution in the error, since it involves what happens near the boundary. Referring to Step 2 and Step 3 of the algorithm, one easily obtains

$$
\begin{aligned}
B_{i}= & \mathbb{E}_{x}\left(\mathbf{1}_{X_{t_{i}}^{N} \in V_{\partial D}(R)}\left[v\left(t_{i+1}, Y_{t_{i+1}}^{i}\right)-v\left(t_{i}, X_{t_{i}}^{N}\right)-h\left(\pi_{\partial D}^{\gamma}\left(X_{t_{i}}^{N}\right)\right)\left(k_{t_{i+1}}^{N}-k_{t_{i}}^{N}\right)\right]\right) \\
& +\mathbb{E}_{x}\left(\mathbf{1}_{X_{t_{i}}^{N} \in V_{\partial D}(R)}\left[v\left(t_{i+1}, \pi_{D}^{\gamma}\left(Y_{t_{i+1}}^{i}\right)\right)-v\left(t_{i+1}, Y_{t_{i+1}}^{i}\right)-h\left(\pi_{\partial D}^{\gamma}\left(X_{t_{i}}^{N}\right)\right)\left(F^{\gamma}\right)^{-}\left(Y_{t_{i+1}}^{i}\right)\right]\right)+O_{\mathrm{pol}}(N) \\
:= & C_{i}+D_{i}+O_{\mathrm{pol}}(N),
\end{aligned}
$$

where we put for $t \in\left[t_{i}, t_{i+1}\right], Y_{t}^{i}=X_{t_{i}}^{N}+B\left(X_{t_{i}}^{N}\right)\left(t-t_{i}\right)+\sigma\left(X_{t_{i}}^{N}\right)\left(W_{t}-W_{t_{i}}\right)+\gamma\left(\pi_{\partial D}^{\gamma}\left(X_{t_{i}}^{N}\right)\right)$ $\left(k_{t}^{N}-k_{t_{i}}^{N}\right)$, piecewise diffusion process which is reflected in $D\left(\pi_{\partial D}^{\gamma}\left(X_{t_{i}}^{N}\right)\right)=\left\{z \in \mathbb{R}^{d}:\left(z-\pi_{\partial D}^{\gamma}\left(X_{t_{i}}^{N}\right)\right) \cdot n\left(\pi_{\partial D}^{\gamma}\right.\right.$ $\left.\left.\left(X_{t_{i}}^{N}\right)\right)>0\right\}$.

Theorems 3.2 and 3.3 are proved if one shows that

$$
\begin{array}{ll}
\sum_{i=0}^{N-1} C_{i}= \begin{cases}O\left(N^{-1}\right) & \text { in the conormal case } \\
O\left(N^{-1 / 2}\right) & \text { in the general case }\end{cases} \\
\sum_{i=0}^{N-1} D_{i}=O\left(N^{-1}\right) & \text { in the general case. }
\end{array}
$$

For this, we divide computations in several steps.

\section{Step 1. Estimation of the term $C_{i}$ in the general case}

For $y \in V_{\partial D}(R) \cap \bar{D}$, set $s_{y}=\pi_{\partial D}^{\gamma}(y)$. Consider $\left(Y_{t}^{i}\right)_{0 \leq t \leq T / N}$ the reflected diffusion in the half-space $D(y)$ :

$$
Y_{t}^{i}=y+B(y) t+\sigma(y) W_{t}+\gamma\left(s_{y}\right) k_{t}^{N} .
$$

Applying Markov property in $C_{i}$, one has to evaluate

$$
\begin{aligned}
\mathbb{E}_{y}\left(v\left(t_{i+1}, Y_{T / N}^{i}\right)-v\left(t_{i}, y\right)-h\left(s_{y}\right) k_{T / N}^{N}\right)= & \int_{0}^{T / N} \mathrm{~d} t \mathbb{E}_{y}\left(\left(\partial_{t}+\mathcal{L}_{y}\right) v\left(t_{i}+t, Y_{t}^{i}\right)\right) \\
& +\mathbb{E}_{y}\left(\int_{0}^{T / N} \mathrm{~d} t\left(\nabla v\left(t_{i}+t, Y_{t}^{i}\right) \cdot \gamma\left(s_{y}\right)-h\left(s_{y}\right)\right) \mathrm{d} k_{t}^{N}\right) \\
:= & C_{i, 1}+C_{i, 2} .
\end{aligned}
$$

Evaluation of $C_{i, 1}$. Apply Ito's formula, remarking that $\left(\partial_{t}+\mathcal{L}_{y}\right) v\left(t_{i}+t, Y_{t}^{i}\right)=0$ for $t=0$; it follows that

$$
\begin{aligned}
C_{i, 1}= & \int_{0}^{T / N} \mathrm{~d} t \int_{0}^{t} \mathrm{~d} r \mathbb{E}_{y}\left(\left(\partial_{t}+\mathcal{L}_{y}\right)^{2} v\left(t_{i}+r, Y_{r}^{i}\right)\right) \\
& +\int_{0}^{T / N} \mathrm{~d} t \mathbb{E}_{y}\left(\int_{0}^{t} \mathrm{~d} r \nabla\left(\partial_{t}+\mathcal{L}_{y}\right) v\left(t_{i}+r, Y_{r}^{i}\right) \cdot \gamma\left(s_{y}\right) \mathrm{d} k_{r}^{N}\right) .
\end{aligned}
$$


The first term in the r.h.s. of (53) has obviously the expected order $K(T) N^{-2}$. For the second, apply formula (45) to $\left(Y_{t}^{i}\right)_{0 \leq t \leq T / N}$ defined in (50) to obtain:

$$
\begin{aligned}
& \int_{0}^{T / N} \mathrm{~d} t \mathbb{E}_{y}\left(\int_{0}^{t} \mathrm{~d} r \nabla\left(\partial_{t}+\mathcal{L}_{y}\right) v\left(t_{i}+r, Y_{r}^{i}\right) \cdot \gamma\left(s_{y}\right) \mathrm{d} k_{r}^{N}\right) \\
& =\frac{1}{2} a(y) \int_{0}^{T / N} \mathrm{~d} t \int_{0}^{t} \mathrm{~d} r \int_{\partial D(y)} \mathrm{d} s \nabla\left(\partial_{t}+\mathcal{L}_{y}\right) v\left(t_{i}+r, s\right) \cdot \gamma\left(s_{y}\right) p_{r}^{N}(y, s)
\end{aligned}
$$

where $p_{t}^{N}(y, z)$ is the transition density of $\left(Y_{t}^{i}\right)_{0 \leq t \leq T / N}$. Considering (5), the quantity $a(y)$ is defined by $a(y)=\frac{n\left(s_{y}\right) \cdot \sigma \sigma^{*}(y) n\left(s_{y}\right)}{n\left(s_{y}\right) \cdot \gamma\left(s_{y}\right)}$. To evaluate the surface integral, there is no loss of generality to assume that $D(y)=$ $\left\{z \in \mathbb{R}^{d}: z_{1}>0\right\}$ : in that setting, we set $s_{y}=\left[\begin{array}{c}0 \\ s_{y}^{\prime}\end{array}\right] \in \partial D(y)$ for $s_{y}^{\prime} \in \mathbb{R}^{d-1}$. Then, one has

$$
\int_{\partial D(y)} \mathrm{d} s \nabla\left(\partial_{t}+\mathcal{L}_{y}\right) v\left(t_{i}+r, s\right) \cdot \gamma\left(s_{y}\right) p_{r}^{N}(y, s)=\int_{\mathbb{R}^{d-1}} \mathrm{~d} z \varphi(z) p_{r}^{N}\left(y,\left[\begin{array}{l}
0 \\
z
\end{array}\right]\right)
$$

where $\varphi(z)=\nabla\left(\partial_{t}+\mathcal{L}_{y}\right) v\left(t_{i}+r,\left[\begin{array}{l}0 \\ z\end{array}\right]\right) \cdot \gamma\left(s_{y}\right)$. A standard Gaussian upper bound is available for $p_{t}^{N}(y, z)$ :

$$
p_{t}^{N}\left(y,\left[\begin{array}{l}
0 \\
z
\end{array}\right]\right) \leq \frac{K(T)}{t^{d / 2}} \exp \left(-c \frac{\left\|y-\left[\begin{array}{l}
0 \\
z
\end{array}\right]\right\|^{2}}{t}\right) \leq \frac{K(T)}{t^{d / 2}} \exp \left(-c^{\prime} \frac{\left(F^{\gamma}(y)\right)^{2}+\left\|z-s_{y}^{\prime}\right\|^{2}}{t}\right) .
$$

Since $\varphi$ is uniformly bounded, one easily obtains that the surface integral (55) is bounded by $\frac{K(T)}{\sqrt{r}} \exp$ $\left(-c^{\prime} \frac{\left(F^{\gamma}(y)\right)^{2}}{r}\right):$ thus, from $(54)$, it readily follows that

$$
\left|\int_{0}^{T / N} \mathrm{~d} t \mathbb{E}_{y}\left(\int_{0}^{t} \mathrm{~d} r \nabla\left(\partial_{t}+\mathcal{L}_{y}\right) v\left(t_{i}+r, Y_{r}^{i}\right) \cdot \gamma\left(s_{y}\right) \mathrm{d} k_{r}^{N}\right)\right| \leq K(T)\left(\frac{T}{N}\right)^{3 / 2} \exp \left(-c^{\prime} \frac{\left(F^{\gamma}(y)\right)^{2}}{T / N}\right) .
$$

Combining (57) and (53), we clearly see that the contributions of $C_{i, 1}$ in terms $C_{i}$ is bounded by $\sum_{i=0}^{N-1}\left[\frac{K(T)}{N^{2}}+\right.$ $\left.K(T)\left(\frac{T}{N}\right)^{3 / 2} \exp \left(-c^{\prime} \frac{\left(F^{\gamma}\left(X_{t_{i}}^{N}\right)\right)^{2}}{T / N}\right)\right]$. Apply the following lemma, which will be proved at last, to conclude that terms $C_{i, 1}$ globally contributes to the order $N^{-1}$ in any case.

Lemma 3.4. Assume (D), ( $\Gamma),(\mathbf{S})$ and $(\mathbf{E})$. Put $c>0$. There is a function $K(T)$ such that

$$
\frac{T}{N} \sum_{i=0}^{N-1} \mathbb{E}_{x}\left(\exp \left(-c \frac{\left(F^{\gamma}\left(X_{t_{i}}^{N}\right)\right)^{2}}{T / N}\right)\right) \leq \frac{K(T)}{\sqrt{N}}
$$

Evaluation of $C_{i, 2}$. Using the same arguments as before, we rewrite $C_{i, 2}$ using a surface integral:

$$
C_{i, 2}=\frac{1}{2} a(y) \int_{0}^{T / N} \mathrm{~d} t \int_{\partial D(y)} \mathrm{d} s\left(\nabla v\left(t_{i}+t, s\right) \cdot \gamma\left(s_{y}\right)-h\left(s_{y}\right)\right) p_{t}^{N}(y, s) .
$$


We can once again consider w.l.o.g. that $D(y)=\left\{z \in R^{d}: z_{1}>0\right\}$ : thus,

$$
\int_{\partial D(y)} \mathrm{d} s\left(\nabla v\left(t_{i}+t, s\right) \cdot \gamma\left(s_{y}\right)-h\left(s_{y}\right)\right) p_{t}^{N}(y, s)=\int_{\mathbb{R}^{d-1}} \mathrm{~d} z \phi(z) p_{t}^{N}\left(y,\left[\begin{array}{l}
0 \\
z
\end{array}\right]\right),
$$

where $\phi(z)=\nabla v\left(t_{i}+t,\left[\begin{array}{l}0 \\ z\end{array}\right]\right) \cdot \gamma\left(s_{y}\right)-h\left(s_{y}\right)$. The Neumann condition satisfied by $v$ on $\partial D$ tells us that $\phi(z)=0$ at $z=s_{y}^{\prime}$. Hence, Taylor's formula gives $|\phi(z)| \leq K(T)\left\|z-s_{y}^{\prime}\right\|$ and consequently, using the upper bound (56) for $p_{t}^{N}(y, z)$, one gets:

$$
\begin{aligned}
\left|\int_{\partial D(y)} \mathrm{d} s\left(\nabla v\left(t_{i}+t, s\right) \cdot \gamma\left(s_{y}\right)-h\left(s_{y}\right)\right) p_{t}^{N}(y, s)\right| & \leq K(T) \int_{\mathbb{R}^{d-1}} \mathrm{~d} z\left\|z-s_{y}^{\prime}\right\| \frac{K(T)}{t^{d / 2}} \exp \left(-c^{\prime} \frac{\left(F^{\gamma}(y)\right)^{2}+\left\|z-s_{y}^{\prime}\right\|^{2}}{t}\right) \\
& \leq K(T) \exp \left(-c^{\prime} \frac{\left(F^{\gamma}(y)\right)^{2}}{t}\right) .
\end{aligned}
$$

Thus, one has proved that $\left|C_{i, 2}\right| \leq K(T) \frac{T}{N} \exp \left(-c^{\prime} \frac{\left(F^{\gamma}(y)\right)^{2}}{T / N}\right)$. Finally, the application of Lemma 3.4 combined with (58) and (52) proves that $C_{i, 2}$ contributes globally to the order $N^{-1 / 2}$. This completes the proof of (48) in the general case.

\section{Step 2. Improved estimation of the term $C_{i}$ in the conormal case}

The evaluation for $C_{i, 1}$ needs not to be changed since it already gives the expected order $N^{-1}$. To deal with $C_{i, 2}$ and improve the rate of convergence in (48) in the conormal case, remark that it is sufficient to prove that under this assumption, one has

$$
\left|\int_{\mathbb{R}^{d-1}} \mathrm{~d} z \phi(z) p_{t}^{N}\left(y,\left[\begin{array}{l}
0 \\
z
\end{array}\right]\right)\right| \leq K(T) \sqrt{t} \exp \left(-c^{\prime} \frac{\left(F^{\gamma}(y)\right)^{2}}{t}\right),
$$

instead of (59) in the general case. The improvement relies on some symmetry properties of $p_{t}^{N}(y, s)$ for $s \in$ $\partial D(y)$. Actually, the key argument which comes into play is that, in the conormal case, $z \mapsto p_{t}^{N}\left(y,\left[\begin{array}{c}0 \\ s_{y}^{\prime}+z\end{array}\right]\right)$ is merely an even function in each variable $z_{i}, 1 \leq i \leq d-1$ : in fact, this is true if the drift term $B$ is equal zero and the diffusion matrix $\sigma$ is diagonal.

To state a precise formulation, we start from the process $\left(\tilde{Y}_{t}^{\tilde{B}}\right)_{t \geq 0}$, defined as a Brownian motion with constant drift $\tilde{B}$, reflected in $D(y)=\left\{z \in \mathbb{R}^{d}: z_{1}>0\right\}$ in the constant direction $\tilde{\gamma}$ :

$$
\tilde{Y}_{t}^{\tilde{B}}=\tilde{y}+\tilde{B} t+\tilde{W}_{t}+\tilde{\gamma} \tilde{k}_{t}
$$

where $\tilde{W}$ is a standard Brownian motion and $\tilde{k}_{t}=\frac{1}{\tilde{\gamma}_{1}} \max \left(0, \sup _{0 \leq s \leq t}-\left[\tilde{y}+\tilde{B} s+\tilde{W}_{s}\right]_{1}\right)$. We denote by $\tilde{p}_{t}^{\tilde{B}}(\tilde{y}, z)$ its probability transition density (the dependence w.r.t. $\tilde{B}$ will be useful in the following to reduce to the driftless case). Now, consider $U(y)$, an orthogonal matrix with first column equal to $\left(\sigma^{*}(y) e_{1}\right) /\left\|\sigma^{*}(y) e_{1}\right\|$, where $e_{1}=$ $(1,0, \cdots, 0)^{*}$ and choose $\tilde{y}, \quad \tilde{B}, \quad \tilde{\gamma}$ such that $y=\sigma(y) U(y) \tilde{y}, B(y)=\sigma(y) U(y) \tilde{B}$ and $\gamma\left(s_{y}\right)=(\sigma(y) U(y) \tilde{\gamma}) /\|\sigma(y) U(y) \tilde{\gamma}\|$. Then, the process $\left(\sigma(y) U(y) \tilde{Y}_{t}^{\tilde{B}}\right)_{t \geq 0}$ is equal in law to $\left(Y_{t}^{i}\right)_{t \geq 0}$ defined by (50). Indeed, since $[\sigma(y) U(y) V]_{1}=V_{1}$ for any $V \in \mathbb{R}^{d}$, one has

$$
\begin{aligned}
\sigma(y) U(y) \tilde{Y}_{t}^{\tilde{B}} & =\sigma(y) U(y) \tilde{y}+\sigma(y) U(y) \tilde{B} t+\sigma(y) U(y) \tilde{W}_{t}+\frac{\sigma(y) U(y) \tilde{\gamma}}{\tilde{\gamma}_{1}} \max \left(0, \sup _{0 \leq s \leq t}-\left[\tilde{y}+\tilde{B} s+\tilde{W}_{s}\right]_{1}\right) \\
& =y+B(y) t+\sigma(y) \hat{W}_{t}+\frac{\gamma\left(s_{y}\right)}{\gamma_{1}\left(s_{y}\right)} \max \left(0, \sup _{0 \leq s \leq t}-\left[y+B(y) s+\sigma(y) \hat{W}_{s}\right]_{1}\right),
\end{aligned}
$$


where $\left(\hat{W}_{t}=U(y) \tilde{W}_{t}\right)_{t \geq 0}$ defines a new Brownian motion. It follows that

$$
p_{t}^{N}(y, z)=\tilde{p}_{t}^{\tilde{B}}\left(\tilde{y},(\sigma(y) U(y))^{-1} z\right) \frac{1}{\operatorname{det}(\sigma(y) U(y))} .
$$

It remains to get an explicit expression for $\tilde{p}_{t}^{\tilde{B}}(\tilde{y}, z)$; for this, we combine the Girsanov transformation and Lépingle's results $[18,19]$ to write that for any bounded measurable function $g$, one has

$$
\begin{aligned}
& \mathbb{E}_{\tilde{y}}\left(g\left(\tilde{Y}_{t}^{\tilde{B}}\right)\right)= \mathbb{E}\left(g\left(\tilde{y}+\tilde{W}_{t}+\frac{\tilde{\gamma}}{\tilde{\gamma}_{1}}\right) \max \left(0, \sup _{0 \leq s \leq t}-\left[\tilde{y}+\tilde{W}_{s}\right]_{1}\right) \exp \left(\tilde{B} \cdot W_{t}-\frac{1}{2}|\tilde{B}|^{2} t\right)\right) \\
&= \int_{\mathbb{R}^{d}} \mathrm{~d} w \int_{w_{1}^{-}}^{\infty} \mathrm{d} y^{\prime} g\left(\tilde{y}+w+\frac{\tilde{\gamma}}{\tilde{\gamma}_{1}} \max \left(0,-\tilde{y}_{1}+y^{\prime}\right)\right) \exp \left(\tilde{B} \cdot w-\frac{1}{2}|\tilde{B}|^{2} t\right) \\
& \frac{1}{(2 \pi t)^{d / 2}} \exp \left(-\sum_{i=2}^{d} \frac{w_{i}^{2}}{2 t}\right) \frac{2\left(2 y^{\prime}+w_{1}\right)}{t} \exp \left(-\frac{\left(2 y^{\prime}+w_{1}\right)^{2}}{2 t}\right) .
\end{aligned}
$$

To identify the above expression with $\int_{D(y)} \mathrm{d} z g(z) \tilde{p}_{t}^{\tilde{B}}(\tilde{y}, z)$, we split the above integral in two parts: the first one corresponds to the set $\left\{w \in \mathbb{R}^{d}, w_{1}^{-} \leq y^{\prime} \leq y_{1}\right\}$, for which one puts the change of variables $z=\tilde{y}+w$; for the second one associated to $\left\{w \in \mathbb{R}^{d}, \max \left(w_{1}^{-}, y_{1}\right) \leq y^{\prime}\right\}$, one sets $z=\tilde{y}+w+\frac{\tilde{\gamma}}{\tilde{\gamma}_{1}}\left(-\tilde{y}_{1}+y^{\prime}\right)$ for fixed $y^{\prime}$. These easy computations lead to

$$
\begin{aligned}
\tilde{p}_{t}^{\tilde{B}}(\tilde{y}, z)= & \left.\frac{1}{(2 \pi t)^{d / 2}} \exp \left(-\sum_{i=2}^{d} \frac{[z-\tilde{y}]_{i}^{2}}{2 t}\right)\left(\exp \left(-\frac{[z-\tilde{y}]_{1}^{2}}{2 t}\right)-\exp \left(-\frac{[z+\tilde{y}]_{1}^{2}}{2 t}\right)\right) \exp \left(\tilde{B} \cdot(z-\tilde{y})-\frac{1}{2}|\tilde{B}|^{2} t\right)\right) \\
& +\frac{2}{(2 \pi t)^{d / 2}} \int_{\tilde{y}_{1}}^{\infty} \mathrm{d} y^{\prime} \exp \left(-\sum_{i=2}^{d} \frac{\left[z-\tilde{y}-\frac{\tilde{\gamma}}{\tilde{\gamma}_{1}}\left(-\tilde{y}_{1}+y^{\prime}\right)\right]_{i}^{2}}{2 t}\right) \frac{\left(y^{\prime}+z_{1}\right)}{t} \exp \left(-\frac{\left(y^{\prime}+z_{1}\right)^{2}}{2 t}\right) \\
& \left.\times \exp \left(\tilde{B} \cdot\left(z-\tilde{y}-\frac{\tilde{\gamma}}{\tilde{\gamma}_{1}}\left(-\tilde{y}_{1}+y^{\prime}\right)\right)-\frac{1}{2}|\tilde{B}|^{2} t\right)\right) .
\end{aligned}
$$

Now, denote by $J$ the linear application from $\mathbb{R}^{d-1}$ into $\mathbb{R}^{d-1}$ defined by $(\sigma(y) U(y))^{-1}\left[\begin{array}{l}0 \\ z\end{array}\right]=\left[\begin{array}{c}0 \\ J(z)\end{array}\right]$ : since $\sigma(y) U(y)$ is invertible, $J$ satisfies the same properties. From (61), one deduces that

$$
\begin{aligned}
\int_{\mathbb{R}^{d-1}} \mathrm{~d} z \phi(z) p_{t}^{N}\left(y,\left[\begin{array}{l}
0 \\
z
\end{array}\right]\right)= & \int_{\mathbb{R}^{d-1}} \mathrm{~d} z \phi(z) \tilde{p}_{t}^{\tilde{B}}\left(\tilde{y},(\sigma(y) U(y))^{-1}\left[\begin{array}{l}
0 \\
z
\end{array}\right]\right) \frac{1}{\operatorname{det}(\sigma(y) U(y))} \\
= & \int_{\mathbb{R}^{d-1}} \mathrm{~d} z \phi(z)\left(\tilde{p}_{t}^{\tilde{B}}-\tilde{p}_{t}^{0}\right)\left(\tilde{y},(\sigma(y) U(y))^{-1}\left[\begin{array}{l}
0 \\
z
\end{array}\right]\right) \frac{1}{\operatorname{det}(\sigma(y) U(y))} \\
& +\int_{\mathbb{R}^{d-1}} \mathrm{~d} z^{\prime} \phi\left(J^{-1}\left(z^{\prime}\right)+s_{y}^{\prime}\right) \tilde{p}_{t}^{0}\left(\tilde{y},\left[\begin{array}{c}
0 \\
z^{\prime}+J\left(s_{y}^{\prime}\right)
\end{array}\right]\right) \frac{|\operatorname{det}|}{\operatorname{det}(\sigma(y) U(y))} \\
:= & C_{i, 3}+C_{i, 4} .
\end{aligned}
$$

From the explicit expression of $\tilde{p}_{t}^{\tilde{B}}$, it is easy to check that $\left|\tilde{p}_{t}^{\tilde{B}}(\tilde{y}, z)-\tilde{p}_{t}^{0}(\tilde{y}, z)\right| \leq \frac{K(T)}{t^{(d-1) / 2}} \exp \left(-c \frac{\|z-\tilde{y}\|^{2}}{t}\right)$, for some uniform constants in $t, \tilde{y}, z$. This estimate is sufficient to assert that $\left|C_{i, 3}\right| \leq K(T) \sqrt{t} \exp \left(-c^{\prime} \frac{\left(F^{\gamma}(y)\right)^{2}}{t}\right)$, applying the same arguments than for (59). 
The control of $C_{i, 4}$ requires a more careful analysis of $p_{t}^{0}\left(\tilde{y},\left[\begin{array}{c}0 \\ z^{\prime}+J\left(s_{y}^{\prime}\right)\end{array}\right]\right)$, which from (62) is equal to

$$
\frac{2}{(2 \pi t)^{d / 2}} \int_{\tilde{y}_{1}}^{\infty} d y^{\prime} \exp \left(-\sum_{i=2}^{d} \frac{\left(\left[z^{\prime}+J\left(s_{y}^{\prime}\right)\right]-\tilde{y}-\frac{\tilde{\gamma}}{\tilde{\gamma}_{1}}\left(-\tilde{y}_{1}+y^{\prime}\right)\right]_{i}^{2}}{2 t}\right)^{\frac{y^{\prime}}{t}} \exp \left(-\frac{y^{\prime 2}}{2 t}\right)
$$

Besides, one has $\tilde{y}=(\sigma(y) U(y))^{-1} y=(\sigma(y) U(y))^{-1}\left(s_{y}+F^{\gamma}(y) \gamma\left(s_{y}\right)\right)$, and thus $\tilde{y}_{1}=y_{1}=F^{\gamma}(y) \gamma_{1}\left(s_{y}\right)$ using repeatedly $[\sigma(y) U(y) V]_{1}=V_{1}=\left[(\sigma(y) U(y))^{-1} V\right]_{1}$. Hence, easy computations yield

$$
\begin{aligned}
{\left[\begin{array}{c}
0 \\
z^{\prime}+J\left(s_{y}^{\prime}\right)
\end{array}\right]-\tilde{y}-\frac{\tilde{\gamma}}{\tilde{\gamma}_{1}}\left(-\tilde{y}_{1}+y^{\prime}\right)=} & {\left[\begin{array}{c}
0 \\
z^{\prime}
\end{array}\right]-\frac{(\sigma(y) U(y))^{-1} \gamma\left(s_{y}\right)}{\gamma_{1}\left(s_{y}\right)} y^{\prime} } \\
= & {\left[\begin{array}{c}
0 \\
z^{\prime}
\end{array}\right]-\frac{\left(\sigma\left(s_{y}\right) U\left(s_{y}\right)\right)^{-1} \gamma\left(s_{y}\right)}{\gamma_{1}\left(s_{y}\right)} y^{\prime} } \\
& +\left(\left(\sigma\left(s_{y}\right) U\left(s_{y}\right)\right)^{-1} \gamma\left(s_{y}\right)-(\sigma(y) U(y))^{-1} \gamma\left(s_{y}\right)\right) \frac{y^{\prime}}{\gamma_{1}\left(s_{y}\right)}
\end{aligned}
$$

In our situation where $D(y)=\left\{z_{1}>0\right\}$, the conormal condition at $s_{y}$ states that

$$
\sigma \sigma^{*}\left(s_{y}\right) e_{1}\left\|\gamma\left(s_{y}\right) \Longleftrightarrow\left(\sigma\left(s_{y}\right) U\left(s_{y}\right)\right)^{-1} \gamma\left(s_{y}\right)\right\| e_{1},
$$

so that the term (65) has its $i$-th component $(i \geq 2)$ equal only to $z_{i}^{\prime}$. Writing $\|(\sigma(y) U(y))^{-1} \gamma\left(s_{y}\right)$ $-\left(\sigma\left(s_{y}\right) U\left(s_{y}\right)\right)^{-1} \gamma\left(s_{y}\right) \| \leq C\left|y_{1}\right|$ to control (66), it readily follows, from (64), that

$$
\begin{aligned}
& \left|\tilde{p}_{t}^{0}\left(\tilde{y},\left[\begin{array}{c}
0 \\
z^{\prime}+J\left(s_{y}^{\prime}\right)
\end{array}\right]\right)-\frac{2}{(2 \pi t)^{d / 2}} \exp \left(-\frac{\left\|z^{\prime}\right\|^{2}}{2 t}\right) \exp \left(-\frac{y_{1}^{2}}{2 t}\right)\right| \\
\leq & \frac{K(T)}{t^{(d-1) / 2}} \exp \left(-c \frac{\left\|z^{\prime}\right\|^{2}}{t}\right) \exp \left(-c \frac{\left(F^{\gamma}\right)^{2}(y)}{t}\right) .
\end{aligned}
$$

Thus, one obtains

$$
\begin{aligned}
& \left|C_{i, 4}-\int_{\mathbb{R}^{d-1}} \mathrm{~d} z^{\prime} \phi\left(J^{-1}\left(z^{\prime}\right)+s_{y}^{\prime}\right) \frac{2}{(2 \pi t)^{d / 2}} \exp \left(-\frac{\left\|z^{\prime}\right\|^{2}}{2 t}\right) \exp \left(-\frac{y_{1}^{2}}{2 t}\right) \frac{|\operatorname{det} J|}{\operatorname{det}(\sigma(y) U(y))}\right| \\
\leq & K(T) \sqrt{t} \exp \left(-c^{\prime} \frac{\left(F^{\gamma}(y)\right)^{2}}{t}\right)
\end{aligned}
$$

using the same arguments than for (59). Finally, exploiting the even properties of $\exp \left(-\frac{\left\|z^{\prime}\right\|^{2}}{2 t}\right)$ w.r.t. $z^{\prime}$, one may write

$$
\begin{aligned}
& \left|\int_{\mathbb{R}^{d-1}} \mathrm{~d} z^{\prime} \phi\left(J^{-1}\left(z^{\prime}\right)+s_{y}^{\prime}\right) \frac{2}{(2 \pi t)^{d / 2}} \exp \left(-\frac{\left\|z^{\prime}\right\|^{2}}{2 t}\right) \exp \left(-\frac{y_{1}^{2}}{2 t}\right)\right| \\
= & \left|\int_{\mathbb{R}^{d-1}} \mathrm{~d} z^{\prime}\left(\phi\left(J^{-1}\left(z^{\prime}\right)+s_{y}^{\prime}\right)+\phi\left(J^{-1}\left(-z^{\prime}\right)+s_{y}^{\prime}\right)\right) \frac{1}{(2 \pi t)^{d / 2}} \exp \left(-\frac{\left\|z^{\prime}\right\|^{2}}{2 t}\right) \exp \left(-\frac{y_{1}^{2}}{2 t}\right)\right| \\
\leq & K(T) \sqrt{t} \exp \left(-c^{\prime} \frac{\left(F^{\gamma}(y)\right)^{2}}{t}\right)
\end{aligned}
$$


using that $\left|\phi\left(J^{-1}\left(z^{\prime}\right)+s_{y}^{\prime}\right)+\phi\left(J^{-1}\left(-z^{\prime}\right)+s_{y}^{\prime}\right)\right| \leq K(T)\left\|z^{\prime}\right\|^{2}$ and analogous arguments as above. This proves that $\left|C_{i, 4}\right| \leq K(T) \sqrt{t} \exp \left(-c^{\prime} \frac{\left(F^{\gamma}(y)\right)^{2}}{t}\right)$, and by (63), we have proved (60).

\section{Step 3. Estimation of the term $D_{i}$ in the general case}

Applying Markov property in $D_{i}$ and putting $z=X_{t_{i}}^{N} \in \bar{D} \cap V_{\partial D}(R)$, one has to analyze

$$
\begin{aligned}
& \mathbb{E}\left(v\left(t_{i+1}, \pi_{D}^{\gamma}\left(Y_{T / N}^{i}\right)\right)-v\left(t_{i+1}, Y_{T / N}^{i}\right)-h\left(\pi_{\partial D}^{\gamma}(z)\right)\left(F^{\gamma}\right)^{-}\left(Y_{T / N}^{i}\right) \mid Y_{0}^{i}=z\right) \\
& =\mathbb{E}\left(v\left(t_{i+1}, \pi_{D}^{\gamma}\left(Y_{T / N}^{i}\right)\right)-v\left(t_{i+1}, Y_{T / N}^{i}\right)-h\left(\pi_{\partial D}^{\gamma}\left(Y_{T / N}^{i}\right)\right)\left(F^{\gamma}\right)^{-}\left(Y_{T / N}^{i}\right) \mid Y_{0}^{i}=z\right) \\
& \quad+\mathbb{E}\left(\left[h\left(\pi_{\partial D}^{\gamma}\left(Y_{T / N}^{i}\right)\right)-h\left(\pi_{\partial D}^{\gamma}(z)\right)\right]\left(F^{\gamma}\right)^{-}\left(Y_{T / N}^{i}\right) \mid Y_{0}^{i}=z\right),
\end{aligned}
$$

where $\left(Y_{t}^{i}\right)_{0 \leq t \leq T / N}$ is defined by (50) (with $z$ instead of $y$ ). On the event $\left\{Y_{T / N}^{i} \in D(R)\right\}$, which probability is exponentially close to 1 (apply estimate (15) which is also valid for $\left(Y_{t}^{i}\right)_{0 \leq t \leq T / N}$ ), it is straightforward to see from the definition of $\pi_{D}^{\gamma}($.$) and from the Neumann condition satisfied by v$ on $\partial D$, that one has $\left|v\left(t_{i+1}, \pi_{D}^{\gamma}\left(Y_{T / N}^{i}\right)\right)-v\left(t_{i+1}, Y_{T / N}^{i}\right)-h\left(\pi_{\partial D}^{\gamma}\left(Y_{T / N}^{i}\right)\right)\left(F^{\gamma}\right)^{-}\left(Y_{T / N}^{i}\right)\right| \leq K(T)\left(\left(F^{\gamma}\right)^{-}\right)^{2}\left(Y_{T / N}^{i}\right)$. Inserting this estimate in (67), one easily deduces that

$$
\begin{aligned}
& \left|\mathbb{E}\left(v\left(t_{i+1}, \pi_{D}^{\gamma}\left(Y_{T / N}^{i}\right)\right)-v\left(t_{i+1}, Y_{T / N}^{i}\right)-h\left(\pi_{\partial D}^{\gamma}(z)\right)\left(F^{\gamma}\right)^{-}\left(Y_{T / N}^{i}\right) \mid Y_{0}^{i}=z\right)\right| \\
& \leq K(T) \mathbb{E}\left(\left(\left(F^{\gamma}\right)^{-}\right)^{2}\left(Y_{T / N}^{i}\right)+\left\|Y_{T / N}^{i}-z\right\|\left(F^{\gamma}\right)^{-}\left(Y_{T / N}^{i}\right) \mid Y_{0}^{i}=z\right)+O_{\mathrm{pol}}(N) .
\end{aligned}
$$

To complete the proof of (49), we combine the following upper bounds

$$
\begin{aligned}
\mathbb{E}\left(\left(\left(F^{\gamma}\right)^{-}\right)^{2}\left(Y_{T / N}^{i}\right) \mid Y_{0}^{i}=z\right) & \leq K(T)\left(\frac{T}{N}\right)^{3 / 2} \exp \left(-c \frac{d^{2}(z, \partial D)}{T / N}\right) \\
\mathbb{E}\left(\left\|Y_{T / N}^{i}-z\right\|\left(F^{\gamma}\right)^{-}\left(Y_{T / N}^{i}\right) \mid Y_{0}^{i}=z\right) & \leq K(T)\left(\frac{T}{N}\right)^{3 / 2} \exp \left(-c \frac{d^{2}(z, \partial D)}{T / N}\right)
\end{aligned}
$$

with Lemma 3.4 (recall that $d^{2}(z, \partial D) \geq\left(F^{\gamma}(z)\right)^{2} /\left(c_{1}\right)^{2}$ by Prop. 1.1).

Now, let us prove (68). By definition, the process $\left(Y_{t}^{i}\right)_{0 \leq t \leq T / N}$ takes values in $D(z)$ : using the equivalence between $F^{\gamma}$ and $F^{n}$, one gets

$$
\mathbb{E}\left(\left(\left(F^{\gamma}\right)^{-}\right)^{2}\left(Y_{T / N}^{i}\right) \mid Y_{0}^{i}=z\right) \leq C \mathbb{E}\left(\mathbf{1}_{Y_{T / N}^{i} \in D(z)} \mathbf{1}_{Y_{T / N}^{i} \notin D}\left|F^{n}\right|^{2}\left(Y_{T / N}^{i}\right) \mid Y_{0}^{i}=z\right) .
$$

The remainder of the computations can now follow the routine developed to obtain (23). We briefly recall the main arguments. First, the Gaussian upper bound $\frac{K(T)}{(T / N)^{d / 2}} \exp \left(-c \frac{\|z-y\|^{2}}{T / N}\right)$ for the density $p_{T / N}^{N}(z, y)$ of $Y_{T / N}^{i}$ is still valid, as for $X_{T / N}^{N}$ in (23). Furthermore, the uniform interior sphere condition may be replaced by the uniform interior ellipsoid condition: namely, we consider w.l.o.g. that the new origin is given by $z_{0}=$ $\pi_{\partial D}^{\gamma}(z)+R \gamma\left(\pi_{\partial D}^{\gamma}(z)\right)$ and the new axes are given by $\left(-\gamma\left(\pi_{\partial D}^{\gamma}(z)\right), e_{2}, \cdots, e_{d}\right)$ where $\left(e_{2}, \cdots, e_{d}\right)$ forms an orthonormal basis of the tangent plane to $\partial D$ at $\pi_{\partial D}^{\gamma}(z)$ (see Fig. 6): note that the new coordinates are not orthonormal since $\gamma\left(\pi_{\partial D}^{\gamma}(z)\right)$ is a priori different from $n\left(\pi_{\partial D}^{\gamma}(z)\right)$. In this new coordinate systems, the set $\mathcal{E}\left(z_{0}, R\right)=\left\{y^{\prime} \in \mathbb{R}^{d}:\left\|y^{\prime}-z_{0}\right\| \leq R\right\}$ is an ellipsoid whose intersection with $\partial D$ is restricted to $\pi_{\partial D}^{\gamma}(z)$, whereas the half-space $D(z)$ is given by $D(z)=\left\{y^{\prime} \in \mathbb{R}^{d}: y_{1}^{\prime}<R\right\}$. Considering $\mathcal{E}\left(z_{0}, R\right)$ instead of $B\left(z_{0}, R\right)$ in $(24)$ leads easily to (68) by mimicking the computations.

Same arguments can be used to prove (69) and we omit further details. 


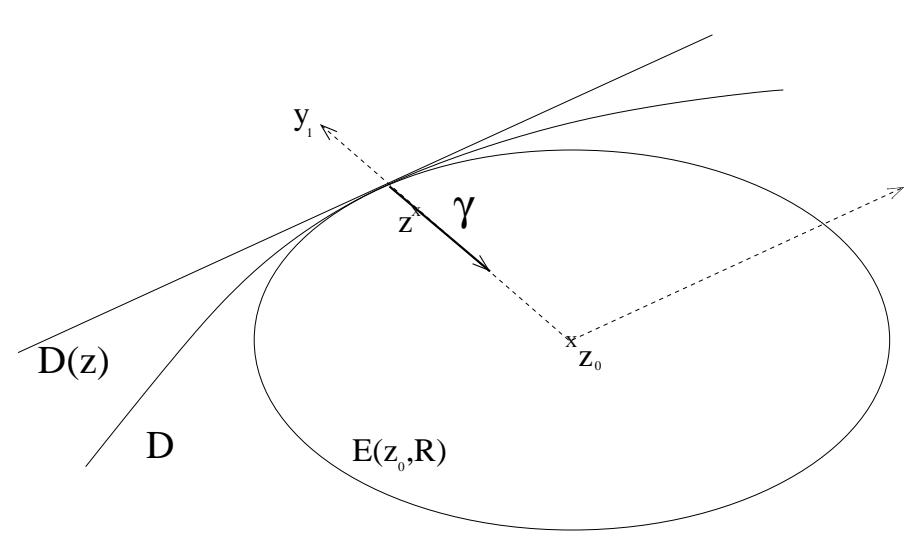

Figure 6. $D$ satisfies the uniform interior ellipsoid condition.

\section{Step 4. Proof of Lemma 3.4}

Put $i \geq 1$ and $t \in\left[t_{i-1}, t_{i}\right)$. For $X_{t_{i-1}}^{N} \in V_{\partial D}(R)$, let us introduce the process $\left(Y_{t}^{i-1}\right)_{t_{i-1} \leq t \leq t_{i}}$ defined as in (50). Conditioning on $\mathcal{F}_{t}$, one has, for some constant $c>0$ changing value from one line to an other:

$$
\begin{aligned}
& \mathbb{E}_{x}\left(\exp \left(-c \frac{\left(F^{\gamma}\left(X_{t_{i}}^{N}\right)\right)^{2}}{T / N}\right)\right) \\
\leq & \mathbb{E}_{x}\left(\mathbf{1}_{X_{t_{i-1}}^{N} \in V_{\partial D}(R)} \mathbb{E}\left(\mathbf{1}_{Y_{t_{i}}^{i-1} \in V_{\partial D}(R) \cap D} \exp \left(-c \frac{\left(F^{\gamma}\left(Y_{t_{i}}^{i-1}\right)\right)^{2}}{T / N}\right)+\mathbf{1}_{Y_{t_{i}}^{i-1} \in V_{\partial D}(R) \cap D^{c}} \mid \mathcal{F}_{t}\right)\right)+O_{\mathrm{pol}}(N) \\
\leq & \mathbb{E}_{x}\left(\mathbf{1}_{X_{t_{i-1}}^{N} \in V_{\partial D}(R)} \mathbb{E}\left(\exp \left(-c \frac{\left(F^{n}\left(Y_{t_{i}}^{i-1}\right)\right)^{2}}{T / N}\right)+\mathbf{1}_{Y_{t_{i}}^{i-1} \notin D^{c}} \mid \mathcal{F}_{t}\right)\right)+O_{\mathrm{pol}}(N) \\
\leq & \mathbb{E}_{x}\left(\mathbf{1}_{X_{t_{i-1}}^{N} \in V_{\partial D}(R)} \mathbf{1}_{Y_{t}^{i-1} \in V_{\partial D}(R)} \exp \left(-c \frac{\left(F^{n}\left(Y_{t}^{i-1}\right)\right)^{2}}{T / N}\right)\right)+O_{\mathrm{pol}}(N) \\
\leq & \mathbb{E}_{x}\left(\mathbf{1}_{X_{t_{i-1}}^{N} \in V_{\partial D}(R)} \mathbf{1}_{Y_{t}^{i-1} \in V_{\partial D}(R) \cap D} \exp \left(-c \frac{\left(F^{n}\left(Y_{t}^{i-1}\right)\right)^{2}}{T / N}\right)\right)+K(T) \sqrt{\frac{T}{N}}+O_{\mathrm{pol}}(N)
\end{aligned}
$$

To prove inequality (71), we used in the conditional expectation, Lemma 2.8 combined with a Gaussian upper bound for the density of $Y_{t_{i-1}}^{i}$ conditionally on $\mathcal{F}_{t}$ to deal with the first term, and for the second one, the estimate (15). Inequality (72) is proved using same kind of arguments that for (23, 68) and (69). Thus, one gets

$$
\begin{aligned}
& \frac{T}{N} \mathbb{E}_{x}\left(\exp \left(-c \frac{\left(F^{\gamma}\left(X_{t_{i}}^{N}\right)\right)^{2}}{T / N}\right)\right) \\
& \leq \int_{t_{i-1}}^{t_{i}} \mathrm{~d} t \mathbb{E}_{x}\left(\mathbf{1}_{X_{t_{i-1}}^{N} \in V_{\partial D}(R)} \mathbb{E}\left(\mathbf{1}_{Y_{t}^{i-1} \in V_{\partial D}(R) \cap D} \exp \left(-c \frac{\left(F^{n}\left(Y_{t}^{i-1}\right)\right)^{2}}{T / N}\right) \mid \mathcal{F}_{t_{i-1}}\right)\right)+O\left(N^{-3 / 2}\right) \\
& \leq \frac{1}{\sigma_{0}^{2}} \int_{0}^{R} \mathrm{~d} y \exp \left(-c \frac{y^{2}}{T / N}\right) \mathbb{E}_{x}\left(\mathbf{1}_{X_{t_{i-1}}^{N} \in V_{\partial D}(R)} \mathbb{E}\left[L_{t_{i-1} \rightarrow t_{i}}^{y}\left(F^{n}\left(Y^{i-1}\right)\right) \mid \mathcal{F}_{t_{i-1}}\right]\right)+O\left(N^{-3 / 2}\right),
\end{aligned}
$$


where we apply for the last inequality the arguments used to deal with (33) (i.e. the occupation times formula to evaluate the time integral). From Tanaka's formula, we deduce that

$$
\begin{aligned}
& \mathbb{E}\left[L_{t_{i-1} \rightarrow t_{i}}^{y}\left(F^{n}\left(Y_{.}^{i-1}\right)\right) \mid \mathcal{F}_{t_{i-1}}\right] \\
= & 2 \mathbb{E}\left[\left(F^{n}\left(Y_{t_{i}}^{i-1}\right)-y\right)^{+}-\left(F^{n}\left(Y_{t_{i-1}}^{i-1}\right)-y\right)^{+}-\int_{t_{i-1}}^{t_{i}} \mathbf{1}_{F^{n}\left(Y_{t}^{i-1}\right)>y} \mathrm{~d}\left(F^{n}\left(Y_{t}^{i-1}\right)\right) \mid \mathcal{F}_{t_{i-1}}\right] \\
= & 2 \mathbb{E}\left[\left(F^{n}\left(Y_{t_{i}}^{i-1}\right)-y\right)^{+}-\left(F^{n}\left(Y_{t_{i-1}}^{i-1}\right)-y\right)^{+} \mid \mathcal{F}_{t_{i-1}}\right] \\
& -2 \mathbb{E}\left[\int_{t_{i-1}}^{t_{i}} \mathrm{~d} t \mathbf{1}_{F^{n}\left(Y_{t}^{i-1}\right)>y} \nabla F^{n}\left(Y_{t}^{i-1}\right) \cdot \gamma\left(Y_{t_{i-1}}^{i-1}\right) \mathrm{d} k_{t}^{N} \mid \mathcal{F}_{t_{i-1}}\right]+O\left(\frac{T}{N}\right) .
\end{aligned}
$$

Assumption $(\Gamma)$ ensures that the integrand in the integral w.r.t. $k_{t}^{N}$ is positive (with probability exponentially close to 1 w.r.t. $N)$. Furthermore, obviously for $y \geq 0$, one has $\left(F^{n}\left(Y_{t_{i}}^{i-1}\right)-y\right)^{+}=\left(F^{n}\left(X_{t_{i}}^{N}\right)-y\right)^{+}$. This proves that $\frac{T}{N} \sum_{i=0}^{N-1} \mathbb{E}_{x}\left(\exp \left(-c \frac{\left(F^{\gamma}\left(X_{t_{i}}^{N}\right)\right)^{2}}{T / N}\right)\right)$ is upper bounded by

$$
O\left(N^{-1 / 2}\right)+\frac{2}{\sigma_{0}^{2}} \int_{0}^{R} \mathrm{~d} y \exp \left(-c \frac{y^{2}}{T / N}\right) \sum_{i=1}^{N-1} \mathbb{E}_{x}\left[\left(F^{n}\left(X_{t_{i}}^{N}\right)-y\right)^{+}-\left(F^{n}\left(X_{t_{i-1}}^{N}\right)-y\right)^{+}\right]=O\left(N^{-1 / 2}\right)
$$

since the sum is telescoping. Lemma 3.4 is proved.

\subsubsection{Proof of Theorem 3.2 in the case of irregular functions $f$}

As for the problem of killed diffusion, when $f$ satisfies $(\mathbf{H 1})$, uniform bound on the derivatives of $v(t, x)$ are not more available and we shall use the following result, which is analogous to Lemma 2.7. The proof uses the same arguments and we omit it.

Lemma 3.5. Assume (D), (Г), (S), (E) and that $f$ satisfies (H1). Then, for all multi-index $\alpha$, for all $g \in C_{b}^{|\alpha|}\left(\mathbb{R}^{d}, \mathbb{R}\right)$, there is a function $K(T)$ (depending on $\|g\|_{C_{b}^{|\alpha|}}$ ), such that

$$
\begin{aligned}
\forall(s, x) \in[0, T) \times V_{\partial D}(\epsilon / 2) & \left|\partial_{x}^{\alpha} v(s, x)\right| \leq K(T) \frac{\|f\|_{\infty}}{1 \wedge \epsilon^{|\alpha|}} \\
\forall(s, t) \in[0, T) \times[0, T] \quad\left|\mathbb{E}_{x}\left[g\left(Y_{t}\right) \partial_{x}^{\alpha} v\left(s, Y_{s}\right)\right]\right| & \leq \frac{\|f\|_{\infty}}{1 \wedge \epsilon^{|\alpha|}} \frac{K(T)}{T^{\frac{|\alpha|}{2}}}
\end{aligned}
$$

where the process $\left(Y_{t}\right)_{t \geq 0}$ is the piecewise Euler scheme without reflection if $X_{\varphi(t)}^{N} \notin V_{\partial D}(R)$ (see Case a) of the algorithm), or with reflection in the appropriate half-space (see Case b) of the algorithm).

\subsection{Numerical experiments}

We borrow the following test to [7]. We consider a 2-dimensional normally reflected Brownian motion $\left(X_{t}\right)_{0 \leq t \leq 1}$ in the unit sphere and we focus on the quantity $v(t, x)=\mathbb{E}_{x}\left(k_{1-t}+\frac{1}{2}\left\|X_{1-t}\right\|^{2}\right)$. It is easy to check from the PDE solved by $v$ that one has $v(t, x)=1-t+\frac{1}{2}\|x\|^{2}$. Figure 7 represents the results corresponding to the computation of the solution for $x=0$ and $t=0$, with $M=1000000$ simulations. It appears on this example that the procedure based on half space approximation converges very quickly compared to the standard projected Euler scheme. Moreover, the additional complexity (see the tabular below corresponding to $N=10$ discretization times and $M=1000000$ simulations) is less than half more. 


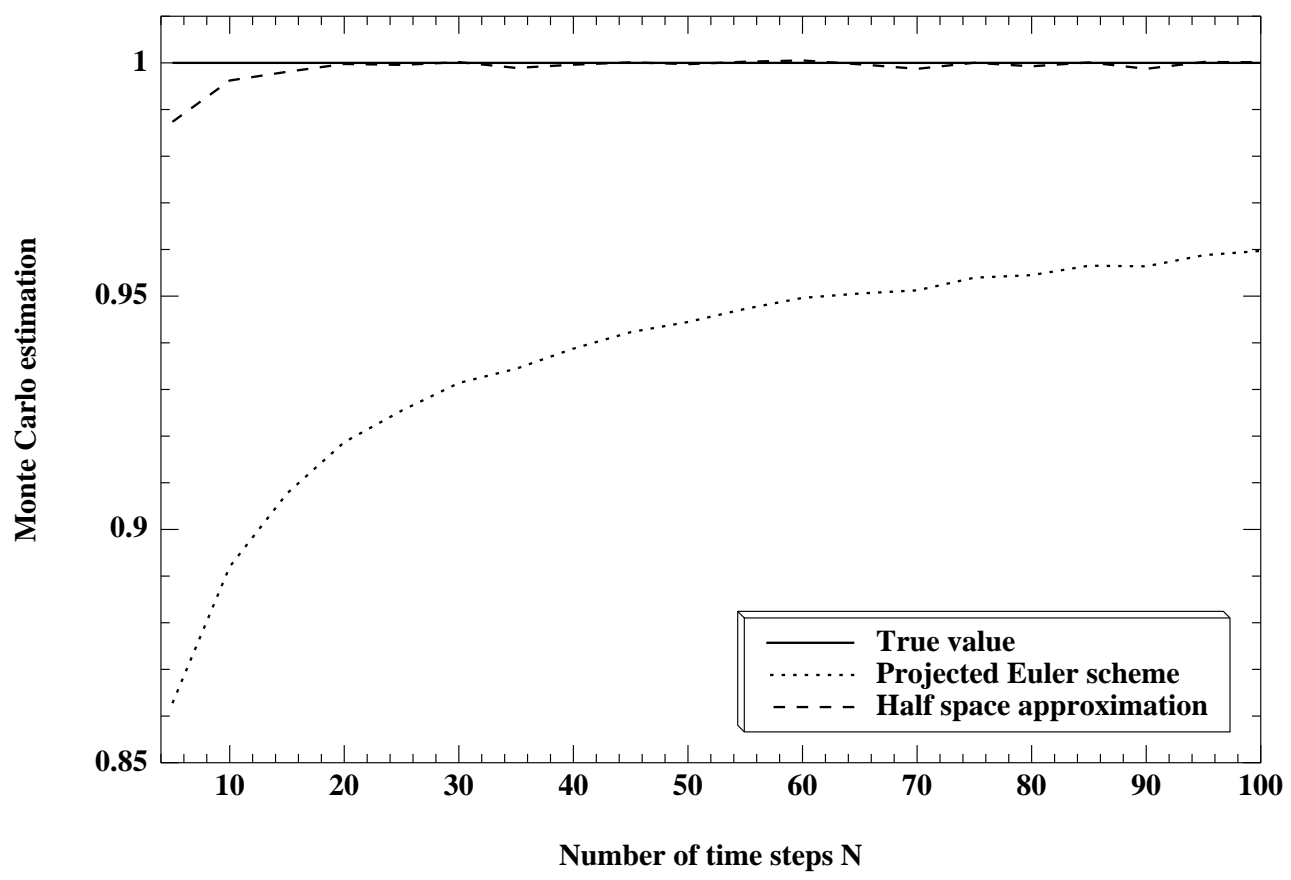

FiguRE 7. Comparison of convergence.

\begin{tabular}{|c|c|c|}
\hline & Projected Euler scheme & Half-space approximation \\
\hline \hline Estimated value & 0.891312 & 0.996198 \\
\hline Relative error & $11 \%$ & $0.4 \%$ \\
\hline Time & $16.37 \mathrm{~s}$ & $23.20 \mathrm{~s}$ \\
\hline
\end{tabular}

We can also discuss the dependence of the error w.r.t the initial value of the process: indeed, the main source error may come somehow from the behavior near the boundary, and by starting close to $\partial D$, one may obtain larger errors. In Figure 8, one has plotted the algebraic error w.r.t. $x=\left(x_{1}, x_{2}\right)$. One can see that the error for the algorithm that we have studied is indeed larger near $\partial D$ than in $x=0$ : note that an analogous behavior occurs for the projected scheme, but with bigger effects.

To continue the test of the accuracy of our procedure, we choose a case where the reflection is not conormal. Namely, we consider the 2-dimensional diffusion process $\left(X_{t}\right)_{0 \leq t \leq T}$ with coefficients defined in (37), with normal reflection in the unit sphere: $D=\left\{x \in \mathbb{R}^{2}:\|x\|<1\right\}$. We set $X_{0}=0$ and $T=1$ and we simply focus on the computation of the expectation of the local time. In Figure 9, we plot the errors w.r.t. $N$ (for $8 \leq N \leq 256$ ) in $\log$ scale, for $M=1000000$ simulations. The linear regression provides an order of convergence estimated to $\alpha=0.42153$ for the Euler scheme with projection, and $\alpha=0.93334$ for our procedure. The conclusion is that even if we have asserted that the order of convergence is at most equal to 0.5 in the case of non conormal reflection, it can be much better in practice.

\section{Conclusion}

In this paper, we have proposed two new numerical schemes for killed and reflected SDEs $\left(X_{t}\right)_{0 \leq t \leq T}$, using an Euler scheme with step time $T / N$ combined with local approximation of the domain into half-space. 

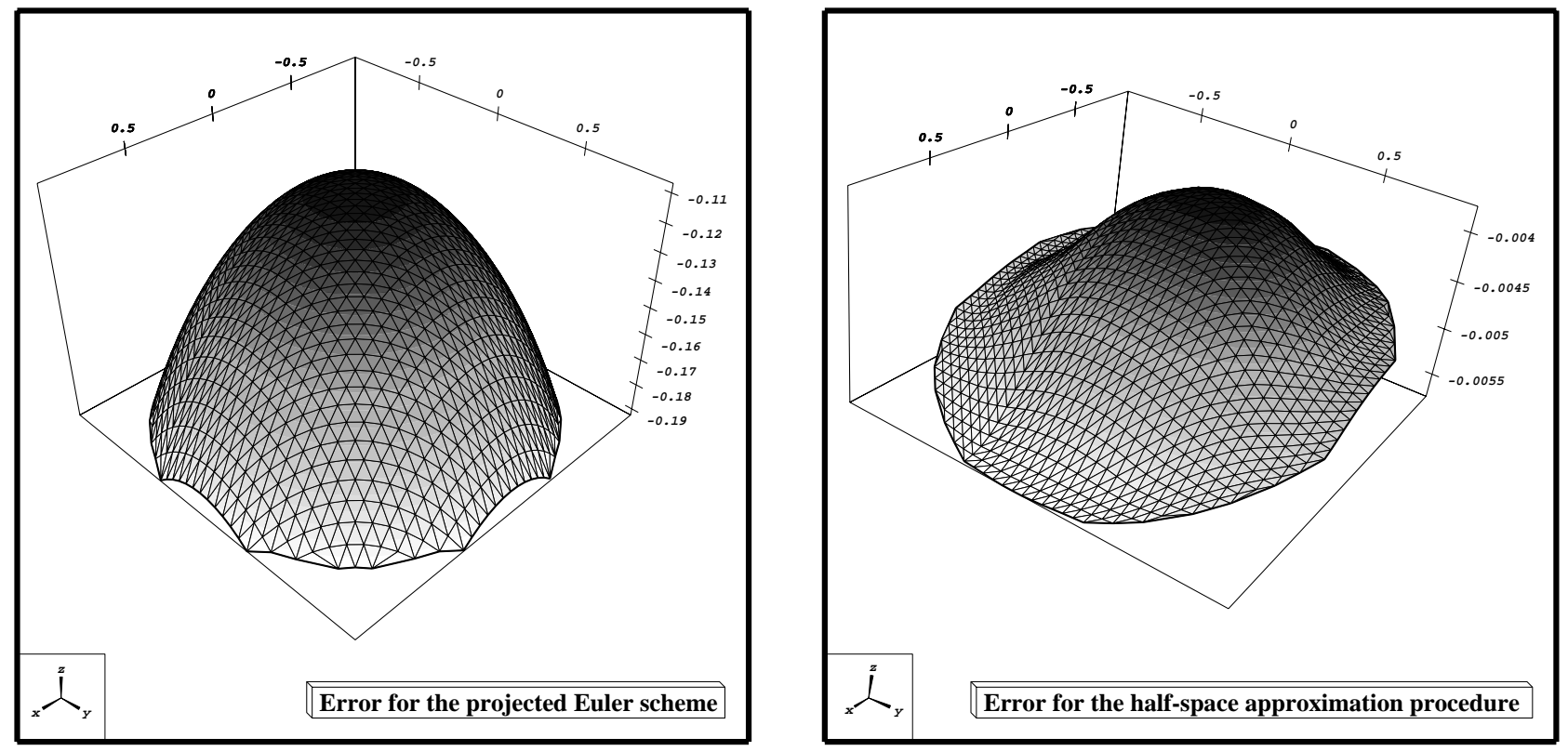

FiguRE 8. Dependence of errors w.r.t. the initial value $x=\left(x_{1}, x_{2}\right)$.

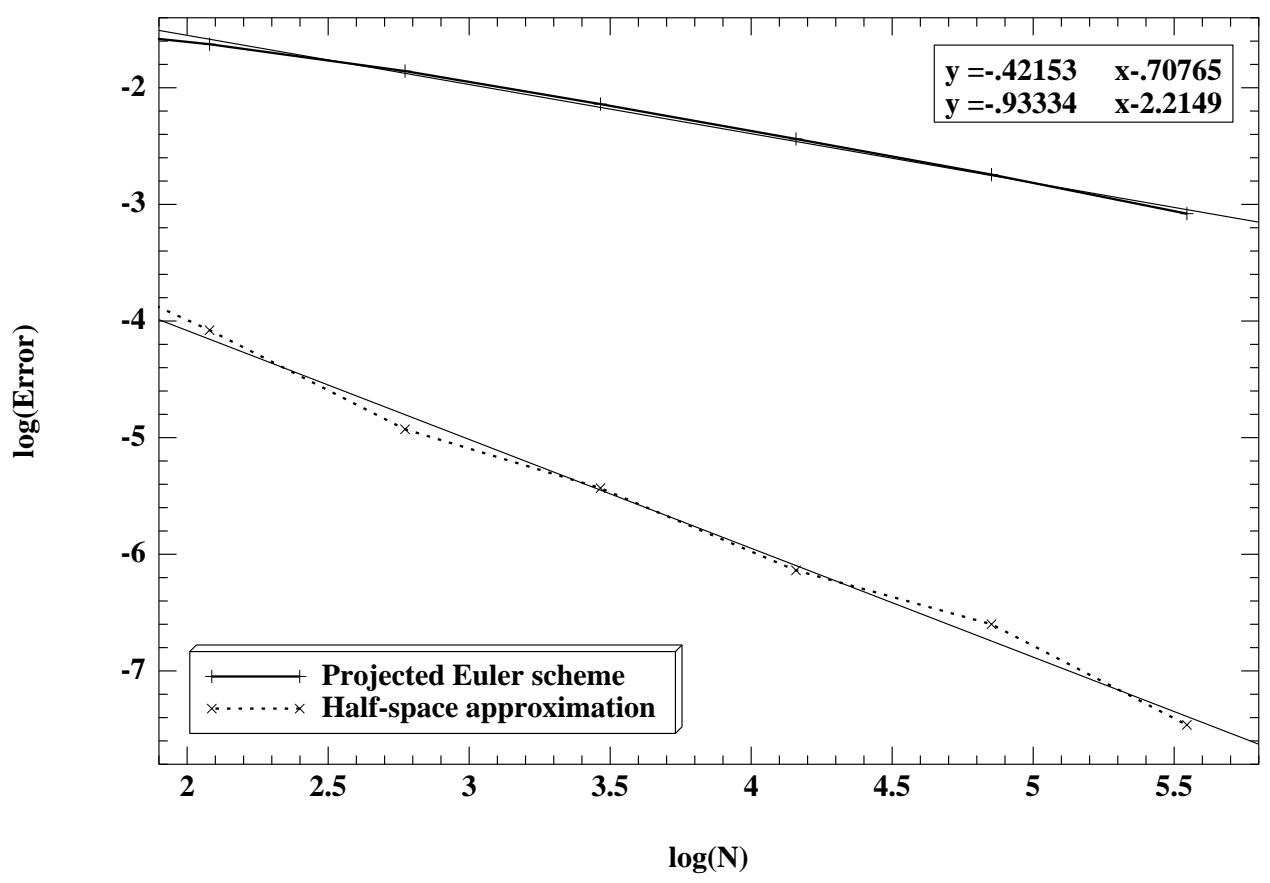

FiguRE 9. Empirical rate of convergence in the non conormal case. 
For diffusions killed at the boundary, the simulation procedure is fully implementable and provides a weak error of order $N^{-1}$. For obliquely reflected diffusions, the rate of convergence is still $N^{-1}$, under the assumption that the reflecting direction is the conormal one. The numerical procedure is also straightforward to implement.

Few extensions. Assumptions on the smoothness of $B, \sigma, D, \gamma, h$ considered in the paper are not the weakest ones, they can be slightly improved: the key point is to ensure enough regularity for the underlying PDE and enough control on the derivatives, and this holds true for Hölder class of functions (see [17]). As instance, we could allow $B, \sigma, D$ to be of class $C^{l}$ for $l \in(4,5)$ instead of $C^{5}$.

The extension of the results to time dependent coefficients $B, \sigma, \gamma, h$ is available as well. For the simulation procedure, one has to adapt the Euler schemes to that situation, by freezing the time $t \in\left[t_{i}, t_{i+1}\right)$ to $t_{i}$ on each interval, this is a classical fact. If we allow $C^{2}$ type smoothness for the involved time dependent functions and adapt the compatibility conditions between $f$ and $h$ for reflected diffusions (see [17]), the proofs go in the same way without additional difficulties.

Open problems. We wish to conclude by listing some open problems which worth being investigated in the future:

1) for SDEs with non conormal reflection, how to get a procedure converging at rate $N^{-1}$ ?

2) how to adapt the simulation algorithm and the analysis to domains with coins?

3 ) when $f$ is measurable (Assumption (H1)) without support condition $(d(\operatorname{Supp}(f), \partial D)>0)$, what is the rate of convergence?

\section{Appendix A. Proof of Proposition 1.1}

Proposition 1.1 brings together more or less well-known results. The case of normal vector field $\gamma=n$ is standard and we refer to [30], or the book by Gilbarg and Trudinger [10] (Appendix, pp. 381-384). For an oblique vector field $\gamma$, we have not found any reference for a proof: hence, we outline the main ideas, following some arguments from [10].

Assertions i), ii), iii), iv). Take $s \in \partial D$ and consider $g^{s}$, a mapping of $\partial D$ in a neighborhood of $s$, i.e. a $C^{5}$ function from $U^{s} \subset \mathbb{R}^{d-1}$ into $\partial D$ : set $z_{0}$ such that $g^{s}\left(z_{0}\right)=s$. Put

$$
G^{s}:\left\{\begin{array}{ccc}
U^{s} \times \mathbb{R} & \longrightarrow & \mathbb{R}^{d} \\
(z, \lambda):=\left(z_{1}, z_{2}, \cdots, z_{d-1}, \lambda\right) & \longmapsto & g^{s}(z)+\lambda \gamma\left(g^{s}(z)\right)
\end{array}\right.
$$

which defines a $C^{4}$ function. Its Jacobian matrix is given by

$$
J^{s}(z, \lambda)=\left[\begin{array}{llll}
\partial_{1} g^{s}(z)+\lambda \partial_{1}\left[\gamma\left(g^{s}(z)\right)\right] & \cdots & \partial_{d-1} g^{s}(z)+\lambda \partial_{d-1}\left[\gamma\left(g^{s}(z)\right)\right] & \gamma\left(g^{s}(z)\right)
\end{array}\right] .
$$

$J^{s}\left(z_{0}, 0\right)$ is invertible since its $d-1$ first columns are vectors which span the tangent plane at $s \in \partial D$, and the last one (i.e. $\gamma(s)$ ) is non tangent to $\partial D$ (Assumption $(\Gamma)$ ). By the inverse mapping theorem, $G^{s}$ defines a $C^{4}$ diffeomorphism from $U^{\prime s} \times\left[-R^{s}, R^{s}\right]$ (for some $R^{s}>0$ ) onto $V^{s}=G^{s}\left(U^{\prime s} \times\left[-R^{s}, R^{s}\right]\right)$. Thus, for $x \in V^{s}$, there exists an unique pair $\left(s^{\prime}, \lambda\right) \in \partial D \times\left[-R^{s}, R^{s}\right]$ such that $x=s^{\prime}+\lambda \gamma\left(s^{\prime}\right)$ : indeed, $s^{\prime}=g^{s}\left(\left(G^{s-1}\right)_{1}(x), \cdots,\left(G^{s-1}\right)_{d-1}(x)\right)$, $\lambda=\left(G^{s-1}\right)_{d}(x)$ and we denote $s^{\prime}$ (resp. $\left.\lambda\right)$ by $\pi_{\partial D}^{\gamma}(x)$ (resp. $\left.F^{\gamma}(x)\right)$.

The compactness assumption on the boundary $\partial D$ enables to consider a finite number of $\left(G^{s_{i}}, U^{\prime s_{i}}, V^{s_{i}}\right)_{1 \leq i \leq k}$ such that the definitions of $\pi_{\partial D}^{\gamma}(x)$ and $F^{\gamma}(x)$ are valid for any $x \in V_{\partial D}(R) \subset \cup_{i=1}^{k} V^{s_{i}}$ for some $R>0$.

The extension of $\pi_{\partial D}^{\gamma}(x)$ and $F^{\gamma}(x)$ in smooth functions on $\mathbb{R}^{d}$ follows from classical arguments (see Sect. IV.4 [17]). We have essentially proved Assertions i) ii) iii) iv). 
Assertion v). It is sufficient to prove the equivalence between $F^{n}$ and $F^{\gamma}$ on $V_{\partial D}(R)$. Hence, let $x \in V_{\partial D}(R)$. Obviously, $\left|F^{\gamma}(x)\right|=\left\|x-\pi_{\partial D}^{\gamma}(x)\right\| \geq d(x, \partial D)=\left|F^{n}(x)\right|$. On the other hand, one has

$$
\begin{aligned}
F^{n}(x) & =\left(x-\pi_{\partial D}^{n}(x)\right) \cdot n\left(\pi_{\partial D}^{n}(x)\right)=\left(x-\pi_{\partial D}^{\gamma}(x)\right) \cdot n\left(\pi_{\partial D}^{n}(x)\right)+\left(\pi_{\partial D}^{\gamma}(x)-\pi_{\partial D}^{n}(x)\right) \cdot n\left(\pi_{\partial D}^{n}(x)\right) \\
& =F^{\gamma}(x) \gamma\left(\pi_{\partial D}^{\gamma}(x)\right) \cdot n\left(\pi_{\partial D}^{n}(x)\right)+\left(\pi_{\partial D}^{\gamma}(x)-\pi_{\partial D}^{n}(x)\right) \cdot n\left(\pi_{\partial D}^{n}(x)\right) .
\end{aligned}
$$

One clearly has $\left\|\pi_{\partial D}^{\gamma}(x)-\pi_{\partial D}^{n}(x)\right\|=\left\|\pi_{\partial D}^{\gamma}\left(\pi_{\partial D}^{n}(x)+F^{n}(x) n\left(\pi_{\partial D}^{n}(x)\right)\right)-\pi_{\partial D}^{n}(x)\right\| \leq C\left|F^{n}(x)\right|$. Moreover, up to shrinking $R$, one can assume that $\gamma\left(\pi_{\partial D}^{\gamma}(x)\right) \cdot n\left(\pi_{\partial D}^{n}(x)\right) \geq c_{0} / 2$ using Assumption $(\Gamma)$. Consequently, it follows from (73) that $\left|F^{\gamma}(x)\right| \leq \frac{2(1+C)}{c_{0}}\left|F^{n}(x)\right|$, completing the proof of $\left.\mathrm{v}\right)$.

Assertion vi). For $s \in \partial D$ (with $z_{0}$ such that $\left.g^{s}\left(z_{0}\right)=s\right)$, one has

$$
J^{s}\left(z_{0}, 0\right)=\left[\begin{array}{llll}
\partial_{1} g^{s}\left(z_{0}\right) & \cdots & \partial_{d-1} g^{s}\left(z_{0}\right) & \gamma\left(g^{s}\left(z_{0}\right)\right)
\end{array}\right],
$$

where $n\left(g^{s}\left(z_{0}\right)\right) \cdot \partial_{i} g^{s}\left(z_{0}\right)=0$ for $i=1, \cdots, d-1$. The inverse mapping theorem ensures that $\nabla F^{\gamma}(s)=$ $\nabla\left(G^{s-1}\right)_{d}(s)$ satisfies $\left(\nabla F^{\gamma}\right)^{*}(s) J^{s}\left(z_{0}, 0\right)=\left(\begin{array}{llll}0 & \cdots & 0 & 1\end{array}\right)$ : it is now easy to check that $\nabla F^{\gamma}(s)=\frac{n}{n \cdot \gamma}(s)$ works.

\section{REFERENCES}

[1] P. Baldi, Exact asymptotics for the probability of exit from a domain and applications to simulation. Ann. Probab. 23 (1995) 1644-1670.

[2] P. Baldi, L. Caramellino and M.G. Iovino, Pricing complex barrier options with general features using sharp large deviation estimates, edited by Niederreiter, Harald et al., Monte-Carlo and quasi-Monte-Carlo methods 1998, in Proc. of a conference held at the Claremont Graduate University. Claremont, CA, USA, June 22-26, 1998. Springer, Berlin (2000) 149-162.

[3] V. Bally and D. Talay, The law of the Euler scheme for stochastic differential equations: I. Convergence rate of the distribution function. Probab. Theory Related Fields 104-1 (1996) 43-60.

[4] M. Bossy, E. Gobet and D. Talay, Computation of the invariant law of a reflected diffusion process (in preparation).

[5] P. Cattiaux, Hypoellipticité et hypoellipticité partielle pour les diffusions avec une condition frontière. Ann. Inst. H. Poincaré Probab. Statist. 22 (1986) 67-112.

[6] P. Cattiaux, Régularité au bord pour les densités et les densités conditionnelles d'une diffusion réfléchie hypoelliptique. Stochastics 20 (1987) 309-340.

[7] C. Constantini, B. Pacchiarotti and F. Sartoretto, Numerical approximation for functionnals of reflecting diffusion processes. SIAM J. Appl. Math. 58 (1998) 73-102.

[8] O. Faugeras, F. Clément, R. Deriche, R. Keriven, T. Papadopoulo, J. Roberts, T. Viéville, F. Devernay, J. Gomes, G. Hermosillo, P. Kornprobst and D. Lingrand, The inverse EEG and MEG problems: The adjoint state approach. I. The continuous case. Rapport de recherche INRIA No. 3673 (1999).

[9] M. Freidlin, Functional integration and partial differential equations. Ann. of Math. Stud. Princeton University Press (1985).

[10] D. Gilbarg and N.S. Trudinger, Elliptic partial differential equations of second order. Springer Verlag (1977).

[11] E. Gobet, Schémas d'Euler pour diffusion tuée. Application aux options barrière, Ph.D. Thesis. Université Denis Diderot Paris 7 (1998).

[12] E. Gobet, Euler schemes for the weak approximation of killed diffusion. Stochastic Process. Appl. 87 (2000) 167-197.

[13] E. Gobet, Efficient schemes for the weak approximation of reflected diffusions. Monte Carlo Methods Appl. 7 (2001) $193-202$. Monte Carlo and probabilistic methods for partial differential equations. Monte Carlo (2000).

[14] E. Hausenblas, A numerical scheme using excursion theory for simulating stochastic differential equations with reflection and local time at a boundary. Monte Carlo Methods Appl. 6 (2000) 81-103.

[15] S. Kanagawa and Y. Saisho, Strong approximation of reflecting Brownian motion using penalty method and its application to computer simulation. Monte Carlo Methods Appl. 6 (2000) 105-114.

[16] S. Kusuoka and D. Stroock, Applications of the Malliavin calculus I, edited by K. Itô, Stochastic Analysis, in Proc. Taniguchi Internatl. Symp. Katata and Kyoto 1982. Kinokuniya, Tokyo (1984) 271-306.

[17] O.A. Ladyzenskaja, V.A. Solonnikov and N.N. Ural'ceva, Linear and quasi-linear equations of parabolic type. Amer. Math. Soc., Providence, Transl. Math. Monogr. 23 (1968).

[18] D. Lépingle, Un schéma d'Euler pour équations différentielles stochastiques réfléchies. C. R. Acad. Sci. Paris Sér. I Math. 316 (1993) 601-605.

[19] D. Lépingle, Euler scheme for reflected stochastic differential equations. Math. Comput. Simulation 38 (1995) $119-126$.

[20] P.L. Lions and A.S. Sznitman, Stochastic differential equations with reflecting boundary conditions. Comm. Pure Appl. Math. 37 (1984) 511-537. 
[21] Y. Liu, Numerical approaches to reflected diffusion processes. Technical Report (1993).

[22] J.L. Menaldi, Stochastic variational inequality for reflected diffusion. Indiana Univ. Math. J. 32 (1983) $733-744$.

[23] G.N. Milshtein, Application of the numerical integration of stochastic equations for the solution of boundary value problems with Neumann boundary conditions. Theory Probab. Appl. 41 (1996) 170-177.

[24] C. Miranda, Partial differential equations of elliptic type. Springer, New York (1970).

[25] E. Pardoux and R.J. Williams, Symmetric reflected diffusions. Ann. Inst. H. Poincaré Probab. Statist. 30 (1994) 13-62.

[26] R. Pettersson, Approximations for stochastic differential equations with reflecting convex boundaries. Stochastic Process. Appl. 59 (1995) 295-308.

[27] R. Pettersson, Penalization schemes for reflecting stochastic differential equations. Bernoulli 3 (1997) $403-414$.

[28] D. Revuz and M. Yor, Continuous martingales and Brownian motion, 2nd Ed. Springer, Berlin, Grundlehren Math. Wiss. 293 (1994).

[29] Y. Saisho, Stochastic differential equations for multi-dimensional domain with reflecting boundary. Probab. Theory Related Fields 74 (1987) 455-477.

[30] J. Serrin, The problem of Dirichlet for quasilinear elliptic differential equations with many independent variables. Philos. Trans. Roy. Soc. London Ser. A 264 (1969) 413-496.

[31] L. Slomiński, On approximation of solutions of multidimensional SDEs with reflecting boundary conditions. Stochastic Process. Appl. 50 (1994) 197-219.

[32] D. Talay and L. Tubaro, Expansion of the global error for numerical schemes solving stochastic differential equations. Stochastic Anal. Appl. 8-4 (1990) 94-120.

[33] R.J. Williams, Semimartingale reflecting Brownian motions in the orthant, Stochastic networks. Springer, New York (1995) $125-137$. 